\title{
The Exon Junction Complex and intron removal prevents resplicing of mRNA
}

\author{
Brian Joseph ${ }^{1,2}$ and Eric C. Lai ${ }^{1,3}$
}

running title: Exon Junction Complex prevents mRNA resplicing

${ }^{1}$ Department of Developmental Biology, Sloan-Kettering Institute, New York, NY, USA.

${ }^{2}$ Louis V. Gerstner, Jr. Graduate School of Biomedical Sciences, Memorial Sloan Kettering

Cancer Center, New York, NY, USA

${ }^{3}$ Corresponding author.

tel: $212-639-5578$

email: laie@mskcc.org 


\begin{abstract}
Accurate splice site selection is critical for fruitful gene expression. Here, we demonstrate the Drosophila EJC suppresses hundreds of functional cryptic splice sites (SS), even though majority of these bear weak splicing motifs and appear incompetent.

Mechanistically, the EJC directly conceals splicing elements through position-specific recruitment, preventing SS definition. We note that intron removal using strong, canonical SS yields AG|GU signatures at exon-exon junctions. Unexpectedly, we discover that scores of these minimal exon junction sequences are in fact EJC-suppressed $5^{\prime}$ and $3^{\prime}$ recursive SS, and that loss of EJC regulation from such transcripts triggers faulty mRNA resplicing. An important corollary is that intronless cDNA expression constructs from aforementioned targets yield high levels of unanticipated, truncated transcripts generated by resplicing. Consequently, we conclude the EJC has ancestral roles to defend transcriptome fidelity by (1) repressing illegitimate splice sites on pre-mRNAs, and (2) preventing inadvertent activation of such sites on spliced segments.
\end{abstract}




\section{Introduction}

The generation of mature mRNA involves multiple metabolic events, which alter the structure of the messenger ribonucleoprotein particle (mRNP). Since the late 1980s, there has been growing appreciation that one such event, splicing, both modulates mRNP composition and influences downstream metabolic processes. Notably, the Exon Junction Complex (EJC) has emerged as a key, splicing-dependent hub for both mRNA processing and posttranscriptional gene regulation.

The EJC is a multisubunit conglomerate that is deposited in a sequence-independent fashion 24 nt upstream of exon-exon junctions (Boehm and Gehring 2016; Le Hir et al. 2016). Assembly of its three-member core complex begins during splicing, and the first step involves the position-specific deposition of the DEAD-box protein elF4AIII onto RNA by the spliceosome factor CWC22. Next, a heterodimer of MAGOH/Mago Nashi and RBM8A/Y14/Tsunagi binds elF4AIII, stabilizing the complex on RNA. The core EJC complex interacts with multiple peripheral complexes involved in diverse RNA metabolism pathways. These include CASC3/Barentsz, members of the ASAP/PSAP (ACIN1/PNN-RNPS1-SAP18) complexes and other splicing factors, the export factor ALYREF, and the NMD protein UPF3B (Schlautmann and Gehring 2020). Accordingly, EJC dysfunction broadly affects development, disease and cancer (Bonnal et al. 2020).

Curiously, while the EJC is well-conserved, the literature indicates fundamental differences in its requirements between invertebrates and vertebrates (Schlautmann and Gehring 2020). The EJC was first linked to the process of nonsense mediated mRNA decay (Kim et al. 2001; Lykke-Andersen et al. 2001), a process that exploits deposition of the EJC by the spliceosome (Le Hir et al. 2000). Translation removes EJCs from the open reading frame, but the presence of premature termination codons cause EJCs to remain within aberrant 3' UTRs, thereby triggering NMD. However, as introns do not inherently elicit NMD in Drosophila, its pathway does not appear to involve the EJC (Nicholson and Muhlemann 2010). 
The EJC impacts other aspects of the transcript lifecycle, including Polll promoterproximal pause release (Akhtar et al. 2019), mRNA localization (Le Hir et al. 2001; Palacios et al. 2004) and translation (Wiegand et al. 2003). In particular, given central connections between the EJC and the spliceosome (Singh et al. 2012), attention has been paid to splicing-related functions of the EJC. In Drosophila, the EJC positively regulates splicing of long introns, such as mapk (Ashton-Beaucage et al. 2010; Roignant and Treisman 2010), and also activates suboptimal splice sites, such as within piwi (Hayashi et al. 2014; Malone et al. 2014). By contrast, recent analysis of the mammalian EJC shows that many of its direct splicing targets are instead inhibited. In particular, the Gehring and Ule groups found that deposition of the EJC directly prevents usage of spurious exonic 5' and 3' splice sites (Blazquez et al. 2018; Boehm et al. 2018).

With these diverse and often opposing roles in mind, we analyzed the effects of the Drosophila EJC on splicing in greater detail. Although Drosophila melanogaster has one of the best annotated metazoan transcriptomes (Brown et al. 2014; Westholm et al. 2014; Sanfilippo et al. 2017), we unexpectedly detect many hundreds of novel splice junctions upon depletion of core EJC components in a single celltype. As in mammals, the fly EJC protects neighboring introns from cryptic splice site activation by occlusion, and this function is required at weak splice sites and under unusual circumstances including out-of-order splicing and recursive splicing. Surprisingly, we discover that a subset of novel junctions arise from resplicing within transcript segments that have already undergone intron removal. These may reflect an intrinsic requirement of the $\mathrm{EJC}$ to suppress regenerated splice sites that might otherwise undergo recursive splicing. This suggests two distinct stages of EJC protection: (1) suppression of cryptic splice sites during pre-mRNA processing and (2) protection of mRNAs from further resplicing after intron removal. Overall, our findings expand a newly appreciated, ancestral function of the EJC, and emphasize that bypass of this regulatory process via cDNA constructs can have unexpected deleterious consequences. 


\section{Results and Discussion}

\section{EJC depletion leads to activation of spurious junctions}

Recently, Roignant and colleagues reported RNA-seq datasets from S2 cells depleted for core EJC factors elF4AIII, tsu (Y14) and mago (Akhtar et al. 2019). We re-examined these data for splicing defects, and paid particular attention to spurious splice site usage. We utilized MAJIQ to acquire currently unannotated junctions (3606 novel splice sites supported by $\geq 5$ split reads in the aggregate data), of which 1677 were $>2$-fold upregulated in at least one EJC-KD condition. As the three core EJC factors are mutually required for stable EJC association at exon-exon junctions, we might expect these to reveal a set of common molecular defects. Indeed, there was both substantial and significant overlap in novel junctions amongst all three conditions ( $p$-value $<1 \times 10^{-8}$ for three-way overlap), and 876 junctions were elevated in two out of three EJC-KD datasets (Figure 1 - figure supplement 1A). To introduce further stringency, we also filtered for $>2$-fold PSI change in 2/3 EJC depletions, yielding 573 spurious junctions from 386 genes (Figure 1 - figure supplement 1B and Supplementary Table 1). These genes are diverse, with gene ontology (GO) analysis comprising diverse cellular processes including system development and signaling (Supplementary Table 2).

The most frequent spurious junctions involved activation of exonic, alternative $5^{\prime}$ or $3^{\prime}$ SS, followed by novel alternative splicing and intronic SS activation (Figure 1A). These are expected to delete exonic sequence (alternative $5^{\prime}$ or $3^{\prime}$ SS) or insert intronic sequence (intronic SS), relative to canonical mRNA products. We depict straw as an example of aberrant splicing occuring at a constitutive exon-exon junction (Figure 1B). Here, depletion of elF4AIII, tsu and mago, but not lacZ control, all induced high-frequency usage of a novel exonic, alternative 5' SS that joins to the constitutive 3' SS 3248 nt downstream. Importantly, this presumably defective transcript comprises the major isoform in all three core-EJC knockdowns, as it removes $91 \mathrm{nt}$ of coding sequence and is thus out of frame. 
We used rt-PCR to validate de novo splice isoforms in EJC-depleted S2 cells. We selected transcripts with high activation of exonic 5' and 3' SS (PSI > 0.2), such as straw, multiple ankyrin repeats single $\mathrm{KH}$ domain (mask), baboon and eukaryotic translation initiation factor $4 G 1$ (elF4G1), but also evaluated targets with moderate changes $(0.01<P S I<0.05)$ such as Crk oncogene and unkempt. As EJC stabilization during pre-mRNA processing requires eIF4AIII, tsu and mago, but not btz, we utilized knockdown of btz and lacZ as controls (Figure 1

- figure supplement 1C). For all eight amplicons tested, we observed splicing defects only under core-EJC (elF4AIII, tsu and mago) knockdown conditions (Figure 1C). These data provide stringent validation of our annotation of spurious junctions, and highlight a previously unappreciated quality control function of the Drosophila EJC.

\section{The EJC suppresses cryptic exonic 3' SS during pre-mRNA processing}

These alterations in transcript processing were reminiscent of how the human EJC, recruited to exon junctions, directly influences the splicing of neighboring introns (Blazquez et al. 2018; Boehm et al. 2018). Accordingly, we examined the mechanism of EJC-regulated splicing defects in Drosophila. We began by examining transcripts with spurious exonic 3' SS. These represent a majority of de novo events observed in our analysis, and are predicted to cause broad loss of mRNA sequences. Cryptic 3' SS exhibit strong positional bias and cluster specifically around exon junctions (Figure 2A). However, while cryptic 3' SS contain the invariant 3' AG dinucleotide (Figure 2 - figure supplement 1A), quantitative assessment of SS strength indicated broad variation (Figure 2A). In fact, most activated 3' SS in this category are extremely weak and would not normally be considered functionally competent, especially when considering their sheer frequency in the transcriptome at large. Thus, it was important to manipulate these RNA substrates to understand their splicing capacities more directly.

We selected CG7408 as a paradigm: it reproducibly exhibited defective splice isoforms in all core-EJC knockdowns (Figure 2B), but its putative 3' SS is extremely weak (NNSPLICE 
score of 0.29, Figure 2A) and poorly conserved (Figure 2 - figure supplement 1B). We used rt-PCR to validate the expected transcript defects in EJC-depleted cells (Figure 2C), and confirmed 183 nt exon deletion relative to the canonical splice isoform via Sanger sequencing. Our cryptic junction replaces intron 1 , where canonical splicing typically utilizes one of three annotated 3' SS, the dominant of which is stereotypically strong (Figure 2 - figure supplement 1B, NNSPLICE score of 0.91). We then constructed a minigene bearing exons 1-4 of CG7408 (Figure 2D, genomic). When transfected into S2 cells, this reporter recapitulated normal splicing through activation of annotated 3' SS (Figure 2E, genomic). Importantly, a "fully pre-spliced" reporter lacking all introns, i.e., mimicking an mRNA expression construct, yielded a single normal product (Figure 2D-E, mRNA). Thus, pre-processed CG7408 transcripts that cannot recruit $E J C$, also do not undergo further processing. At face value, this appears consistent with the hypothesis that the EJC regulates splicing of flanking introns.

We explored this further by testing for potentially distinct consequences of EJC recruitment to individual CG7408 exon junctions, by removing each intron in turn (Figure 2D $\Delta \mathrm{i} 1, \Delta \mathrm{i} 2$ and $\Delta \mathrm{i} 3)$. These manipulations should only abolish EJC recruitment at individual preprocessed exon junctions. $\Delta \mathrm{i} 1$ only produced the dominant canonical isoform and $\Delta \mathrm{i} 3$ produced the two known canonical isoforms at the same proportions as the genomic construct (Figure 2E). By contrast, pre-removal of intron 2 yielded fully aberrant transcripts (Figure 2E, $\Delta \mathrm{i} 2$ ). These tests emphasize the functional requirement of intron 2 for correct processing of CG7408 and demonstrate that even poor matches to consensus splice sites (i.e., the CG7408 cryptic 3' SS) can be potently activated in the absence of the EJC.

We emphasize that these data support a mechanism in which intron 2 is excised first, and this order is required for the correct definition of the annotated intron $13^{\prime}$ SS (Figure 2G). Out-of-order splicing has been previously observed (Takahara et al. 2002; Drexler et al. 2020), but its relationship to accurate pre-mRNA maturation has generally been unclear. These 
experiments, along with recent work by Gehring and colleagues (Boehm et al. 2018) indicate a requirement for out-of-order splicing for proper mRNA maturation.

How does the EJC inhibit definition of cryptic exonic 3' SS? In human cells, the EJC can directly mask cryptic 3' SS. Based on the close clustering of these sites around the position of EJC recruitment (Figure 2A), we reasoned that the EJC may occlude important features of the 3' SS, such as the branchpoint, polypyrimidine tract or 3' intron junction that base-pairs with the U2 snRNP complex. We tested this hypothesis by separating the cryptic 3' SS on our genomic reporter from the site of EJC recruitment, by inserting a 36 nt spacer (Figure 2D, genomic+Spacer). Unlike the genomic construct, which yields only annotated splice isoforms, the genomic+Spacer variant yielded additional truncated transcripts, consistent with derepression of the cryptic 3' SS (Figure 2 F). Altogether, these data demonstrate the fly EJC aids accurate SS selection during pre-mRNA processing by masking cryptic 3' SS.

\section{The EJC prevents cryptic exonic 5' SS activation during pre-mRNA processing}

We next used analogous strategies to study cryptic exonic 5' SS. These sites represent $\sim 35 \%$ of novel splice junctions upregulated under EJC-depleted conditions and are expected to be deleterious to mRNA processing fidelity. Bioinformatic analysis indicated that cryptic 5' SS share general structural properties with 3' SS, such as clear preference in the vicinity of exon junctions but distribution across a wide range of strengths (Figure 3A, Figure 3 - figure

\section{supplement $1 \mathrm{~A})$.}

We selected CG3632 for mechanistic tests, as core-EJC knockdown data showed activation of a poorly conserved, weak cryptic 5' SS (Figure 3B and Figure 3 - figure supplement 1B-C - NNSPLICE score of 0.54 ) on exon 14. Using rt-PCR and Sanger sequencing, we validated that EJC-depletion induces a defective CG3632 splice isoform lacking $71 \mathrm{nt}$ of coding sequence (Figure $3 \mathbf{C}$ ). 
We hypothesized that the EJC, recruited to the exon $13 / 14$ junction, suppresses the cryptic 5' SS on exon 14 and activates the canonical 5' SS during removal of intron 14 . We tested this using a minigene reporter consisting of exon 14 (containing the cryptic 5' SS) and its immediately flanking introns and exons (Figure 3D, genomic). Expression of this reporter in S2 cells predominantly resulted in the canonical product, but we also observed a minor amount of cryptic 5' SS activation (Figure 3E, genomic). As a negative control, we generated a version lacking both introns (Figure 3D, $\Delta \mathrm{i} 13+14$ ), which produced the expected mRNA (Figure 3E, $\Delta \mathrm{i} 13+14)$. Notably, removal of intron 13 alone (Figure 3D, $\Delta \mathrm{i} 13$ ), mimicking loss of EJC recruitment at the exon 13/14 junction, yielded high levels of cryptic 5' SS activation (Figure 3E, $\Delta \mathrm{i} 13$ ) that were fully suppressed by mutation of the cryptic 5' SS in the $\Delta \mathrm{i} 13$ reporter (Figure 3D-E, $\triangle \mathrm{i} 13+\mathrm{SD}$ mut). Altogether, these data support that deposition of the EJC during premRNA processing suppresses cryptic 5' SS during subsequent intron removal.

\section{The EJC suppresses recursive splice sites}

Given that the EJC suppresses both 5' and 3' SS, a potentially more complex scenario might exist if both types of cryptic splice sites were to be activated in the vicinity of each other. We inspected our catalog of spurious junctions for this possibility, and considered that even modest matches to consensus splice sites (Figure 2A and 3A) might serve as viable candidates for further evaluation. Interestingly, many exon junctions were potentially able to regenerate weak splice sites after intron removal, reminiscent of the process of recursive splicing (RS) (Burnette et al. 2005).

We first investigated a spurious junction within Casein kinase IIß (CkIIß), where coreEJC LOF led to loss of $54 \mathrm{nt}$ of canonical mRNA sequence (Figure 4 - figure supplement 1AB). Assessment of the novel 3' SS on exon 3 revealed that it lacks a polypyrimidine tract and is a poor match to the consensus (Figure 4A). On the surface, the mechanism of cryptic 3' SS activation on $C k l / \beta$ might appear similar to that of $C G 7408$ (Figure 2F, Figure 4 - figure 
supplement 1D, path 2). However, upon examining $C k l l \beta$ for splice sites, we found an additional poor recursive 5' SS at the beginning of exon 3 (Figure 4A). Therefore, we imagined an alternate scenario, whereby dual cryptic 5' and 3' SS might be derepressed upon EJC loss, leading to resplicing (Figure 4 - figure supplement 1D, path 1). Crucially, whether one-step splicing (via alternative splicing) or resplicing (via recursive splicing event), the resulting mRNA products are indistinguishable (Figure 4A). Therefore, we devised reporter tests to clarify the underlying mechanism.

We first used rt-PCR to validate that core-EJC knockdown resulted in substantial activation of a truncated $C k \| / ß$ splice isoform corresponding to RNA-seq data (Figure 4 - figure supplement 1C). We then analyzed a series of splicing minigenes (Figure 4B). Expression of CkII $\beta$ exons 2-4 with all introns present produced a single product with the expected introns spliced out (Figure 4C, genomic). We precisely tested the positional necessity of the EJC at each exon junction by pre-removing each intron (Figure $4 \mathrm{~B}, \Delta \mathrm{i} 2$ and $\Delta \mathrm{i} 3$ ). These reporters also underwent normal splicing (Figure $4 \mathrm{C}, \Delta \mathrm{i} 2$ and $\Delta \mathrm{i} 3$ ), demonstrating that $C k l l \beta$ processing defects were in fact mechanistically distinct from those determined for CG7408. Strikingly, upon testing a construct with both introns pre-removed, we observed a switch to truncated product output, corresponding to activation of the unannotated recursive 5' SS and 3' SS (Figure 4B-C, mRNA). This supports a model where the EJC is required at multiple positions to repress spurious 5' and 3' SS simultaneously (Figure 4 - figure supplement 1D, path 1).

We characterized another instance of dual cryptic splice site within CG31156, albeit of a different flavor. Here, sashimi plots indicate activation of an exonic 5' SS within exon 2 (Figure 4 - figure supplement 2A-B) and we validated this $110 \mathrm{nt}$ deletion isoform using rt-PCR (Figure 4D). Importantly, based on these data alone, it would be reasonable to predict this as a case of alternative cryptic 5' SS activation. However, we noticed that removal of the canonical intron 2 regenerates a putative recursive 3' SS at the exon 2/3 boundary (Figure 4E, Figure 4 figure supplement $\mathbf{2 C}$ ). Therefore, we examined reporters to examine the mechanism 
underlying this unwanted splicing pattern. Expression of the genomic reporter that required intron removal yielded the expected mRNA product (Figure 4F, genomic). Conversely, preremoval of the intron and expression of the mRNA resulted in the truncated re-spliced product (Figure 4F, mRNA). Accordingly, these data again indicate that the EJC represses dual cryptic splice sites during mRNA processing (Figure 4 - figure supplement 2D).

Previous annotations of Drosophila recursive splice sites (RSS) concluded that these hybrid 5'/3' SS are highly conserved, flanked by short cryptic downstream exons, and are highly biased to reside in long introns (mean length $~ 50 \mathrm{~kb}$ ) (Duff et al. 2015; Joseph et al. 2018). While it is possible that recursive splicing aids processing of long introns, it is also conceivable that it is easier to capture RS intermediates within long introns. The examples of cryptic RSS on the CkII $\beta$ and CG31156 transcripts clearly deviate from canonical RSS architectural properties, i.e., they are hosted in short introns and exhibit modest to poor conservation. Moreover, the example of a recursive 3' SS in CG31156 is to our knowledge the first validated instance, and represents a conceptually novel RSS location. Importantly, the relevant AG dinucleotide in the CG31156 3' recursive splice site is not preserved beyond the closest species in the melanogaster subgroup (Figure 4 - figure supplement 2C), and the amino acids encoded by the functional 5' RSS in CkIl $\beta$ diverge with clear wobble patterns (Figure 4A). Thus, these examples of cryptic exonic recursive splicing are functional, but evolutionarily fortuitous.

\section{The EJC protects spliced mRNAs from resplicing}

Since many genes span large genomic regions, cDNA constructs have been a mainstay of directed expression strategies. It is generally expected that these should be effective at inducing gain-of-function conditions, yet cDNA constructs are not typically vetted for proper processing. Our finding of dual cryptic splice sites on transcripts was alarming because in both cases, we observed resplicing on mRNA constructs (Figure 4C and 4F). To reiterate, the EJC 
prevents dual cryptic SS from resplicing on transcript segments that have already undergone intron removal, but such protection will be missing from intronless cDNA copies.

We were keen to assess the breadth of this concept. To do so, we examined the sequence of mRNAs bearing EJC-suppressed cryptic SS, and looked for additional unidentified, complementary SS. Notably, since resplicing would have to map to a canonical junction, we looked for regenerated SS at exon junctions. An initial survey for SS invariant dinucleotide signatures (AG for 3' SS and GT for 5' SS) indicated that 64/118 junctions with cryptic 3' SS and 104/183 junctions with cryptic 5' SS were compatible with resplicing. The fact that over half of both classes of cryptic splicing events were potentially compatible with resplicing might at first glance seem like a tremendous enrichment. However, it does in fact reflect fundamental features of extended consensus splice sequences that basepair with the spliceosome, namely the U1 snRNP and U2AF35 binding sites, respectively (Figure 4G-top). Quantification of these sequences indicated a range of regenerated 5' and 3' SS at exon junctions, with at least 59 junctions resembling strong SS (Figure 4H, NNSPLICE>0.75). However, as several cryptic 5' and 3' SS amongst our validated loci (Figures 1-4) were extremely poor, with functional dual cryptic splice sites in $C k I / \beta$ scoring at only 0.13 and 0.26 (Figure 4A), the functional breadth of this phenomenon is undoubtedly broader. Therefore, we imagined a scenario where a core function of the EJC is to repress splice sites that were regenerated at exon junctions as a consequence of intron removal using canonical splice sites (Figure 4G-bottom).

Nevertheless, as this model cannot be explicitly distinguished from alternative splicing without experimental testing, we selected additional loci for analysis. Therefore, we constructed partial cDNA constructs for three genes, encompassing regions we had validated as subject to EJC-suppression of cryptic splicing (Figure 1C), and selected targets that survey a range of regenerated SS strengths. These include straw, which yields a strong 3' RSS (NNSPLICE score of 0.98 ) after removal of intron 3; elF4G1, which regenerates a moderate 5' RSS (NNSPLICE 
score of 0.64 ) after processing of intron 10; and baboon, which produces an exceptionally poor 3' RSS (NNSPLICE score of 0) after removal of intron 4, bearing only the AG dinucleotide.

In contrast to the endogenous genes which produced a single amplicon, expression of all three cDNA constructs yielded substantial re-spliced products, supporting our view that the EJC prevents activation of dual cryptic SS on mRNAs, including SS regenerated at exon junctions (Figure 4I). Unexpectedly, SS strength did not correlate with levels of resplicing. Indeed, the majority of transcripts from all three reporters were truncated, including from baboon. Furthermore, the elF4G1 reporter yielded three truncated products, suggesting that other sequences may also serve as cryptic SS. As these examples of resplicing occur on coding regions of the transcript, all of them either delete amino acids or generate frameshifts. We conclude that many cDNA constructs are potentially prone to resplicing due to loss of protection afforded by the EJC.

\section{Conserved role for the EJC to repress cryptic splicing and implications for cDNA expression}

Although introns are not essential for gene expression, they play important facilitatory roles by enhancing export and translation in part through recruitment of the EJC during splicing. Subsequently, it was recognized that once deposited, the EJC also promotes accurate gene expression by regulating processing of neighboring introns. Recently, in the mammalian setting, the role of the EJC during pre-mRNA splicing was extended to include suppression of cryptic splice sites (Blazquez et al. 2018; Boehm et al. 2018).

Here we reveal that the fly EJC similarly plays a broad role in direct suppression of cryptic exonic splice sites, owing to its characteristic deposition 20-24 nt upstream of exon-exon junctions. Thus, we now appreciate that concealment and suppression of cryptic splice sites is a conserved EJC activity (Boehm et al. 2018). Importantly, the positional recruitment of the EJC during splicing is conserved and sequence-independent (Le Hir et al. 2000). Thus, we infer this 
function should also be independent of splice site divergence between phyla, as well as splice site strength, and should not require accessory components. In contrast, non-conserved roles of the EJC appear to rely on integration within and diversification of distinct functional networks. For example, while the Upf (Up-frameshift) proteins coordinate NMD across eukaryotes (He and Jacobson 2015), the mechanisms differ. In mammals, NMD is coordinated with intron removal through direct interactions between the EJC and Upf3 (Kim et al. 2001; Le Hir et al. 2001; Lykke-Andersen et al. 2001). However, these interactions are not found in invertebrates, and consequently the invertebrate EJC is not involved in NMD (Nicholson and Muhlemann 2010).

In addition to pre-mRNAs, we show that the EJC also suppresses cryptic splice sites within spliced mRNAs. Although this mechanism cannot be distinguished from alternative splicing (Figure 4A) without further experimentaion, we readily detect resplicing on all cDNA constructs tested. Unexpectedly, while these junctions appear to contain just one cryptic SS, our data indicates that these transcripts contain secondary cryptic splice sites that mediate resplicing. Importantly, we validate that even poor matches to SS consensus motifs are competent for resplicing. Curiously, as all of our demonstrated examples involve a recursive event at either the $5^{\prime}$ or $3^{\prime}$ cryptic SS, our findings broaden a phenomenon that was previously described within long introns (Duff et al. 2015; Joseph et al. 2018). Furthermore, canonical SS sequences that undergo base pairing interactions with U1 snRNA (5' SS) and U2AF35 (3' SS) have motifs AG|GURAGU and YAG|GU (Kielkopf et al. 2001; Kondo et al. 2015). It is noteworthy that core splice site signals contain bases that are compatible with regeneration of splice sites and that these naturally occur proximal to EJC recruitment sites. Accordingly, we propose that an ancestral function for the conserved position of EJC deposition may be to prevent accidental activation of regenerated splice sites.

Finally, our observations of resplicing on cDNAs reflect an essential function for introns in protecting mRNA fidelity. For all tested cases of cDNA resplicing on coding sequences, we note deletions of peptide segments or truncations with loss of domains required for protein 
function (Figure 4 - figure supplement 3A-C). Importantly, these affected targets include essential genes, such as elF4G1 and activin receptor baboon. In the case of baboon, the $54 \mathrm{nt}$ splicing defect leads to a deletion of 18 amino acids (195-212, Figure 4 - figure supplement 3A). For elF4G1, resplicing removes $131 \mathrm{nt}$ of mRNA sequence, alters the open reading frame and leads to protein truncation with loss of the MI and W2 domains (Figure 4 - figure supplement 3B). Finally, resplicing on straw transcripts also alters reading frame by removing $91 \mathrm{nt}$ of mRNA, and is predicted to remove 2/3 Plastocyanin-like domains (Figure 4 - figure supplement $3 \mathbf{C}$ ). Thus, our findings have serious implications for functional genomics as well as community genetic studies (Yu et al. 2011; Wei et al. 2020), where cDNA expression constructs and collections are often employed with little attention paid to mRNA processing. Altogether, our work uncovers an important co-transcriptional function of intron removal and the role of the EJC to protect the transcriptome from unwanted resplicing. 


\section{Materials and Methods}

\section{Bioinformatic analysis}

The core-EJC knockdown RNA-sequencing datasets were previously reported (Akhtar et al. 2019) and obtained from the NCBI Gene Expression Omnibus (GSE92389). Raw sequencing data was mapped to the Drosophila reference genome sequence (BDGP Release 6/dm6) using HISAT2 (Kim et al. 2015) under the default settings. Splice junctions were mapped using the MAJIQ algorithm (2.0) under default conditions (Vaquero-Garcia et al. 2016). Splice graphs and known/novel local splice variants were defined with the MAJIQ Builder using annotations of known genes and splice junctions from Ensembl release 95 and all BAM files. The MAJIQ Quantifier was used to calculate relative abundances (percent selected index - PSI) for all defined junctions. The resulting data was output into tabular format using the Voila function.

A custom R script was written to process all MAJIQ-defined novel junctions relative to the Ensembl gene annotations and identify de novo EJC-suppressed junctions. First, we quantified usage of all novel junctions by mining mapped libraries (BAM files) for high quality junction spanning reads with at least $8 \mathrm{nt}$ of overhang and no mismatches. These counts were normalized to sequencing depth per library. To identify de novo junctions that may be upregulated, we first selected junctions with at least 5 split reads. In order to enrich for de novo junctions that are suppressed by the EJC pathway, we looked for those with $>2$ fold difference in at least 2/3 core-EJC RNAi conditions relative to the lacZ control. To apply further stringency, we also required that the PSI measurements reflect sufficient change between treatment and control conditions. Therefore, we applied an additional filter of PSI fold change $>2$ in at least 2/3 core-EJC RNAi conditions. These criteria produced a total of 573 novel junctions. All junctions are reported in Supplementary Table 1. 
The $5^{\prime}$ and 3 ' ends of these junctions were compared against known gene annotations to characterize splice sites. Exonic 5' and 3' SS reflect sites that mapped on exons while the other end mapped to a canonical splice junction, and the same process was used to define intronic $5^{\prime}$ and 3' SS. de novo cases of alternative splicing reflect junctions that utilized annotated splice sites but represented novel connectivity. Sashimi plots were generated using features available on the Integrative Genomics Viewer (IGV) (Robinson et al. 2011).

We generated a custom pipeline to assess recursive splicing potential (Figure 4). Briefly, we identified transcripts that contained cryptic exonic 5' and 3' splice sites. For these transcripts, we mapped the position of all splice junctions on the mRNA, which could in theory generate the observed splicing defects. We examined sequences directly downstream of relevant splice junctions to identify potential 5 ' recursive splicing and those directly upstream to identify potential 3' recursive splicing.

We calculated splice site strengths using NNSPLICE (https://www.fruitfly.org/seq_tools/splice.html) (Reese et al. 1997). The sequences used for these analyses were obtained from mRNA rather than the genomic context, which may contain intronic sequences as well. To generate nucleotide content plots, splice sites and their indicated flanking sequences were obtained from mRNAs and fed to WebLogo version 2.8.2 (Crooks et al. 2004). The splice sites are centered in these plots.

All custom scripts used in this study are reported on the Lai lab GitHub page.

\section{Constructs and cell culture}

All splicing reporters were cloned into pAC-5.1-V5-His (ThermoFisher Scientific) using compatible restriction sites. We used PCR to amplify minigene splicing reporters from Drosophila genomic DNA, and used site directed mutagenesis to remove specified introns. We used cDNAs to amplify reporters lacking introns. For genes with multiple isoforms (such as 
CG7408), we cloned the dominant fragment. All primers used for generating constructs and mutagenesis have been summarized in Supplementary Table 3.

Transfections were performed using S2-R+ cells cultured in Schneider Drosophila medium with $10 \%$ FBS. Cells were seeded in 6 -well plates at a density of $1 \times 10^{6}$ cells $/ \mathrm{mL}$ and transfected with $200 \mathrm{ng}$ of plasmid using the Effectene transfection kit (Qiagen). Cells were harvested following 3 days of incubation.

\section{Knockdown of EJC factors in S2 cells}

The indicated EJC components were knocked down via RNAi (dsRNA-mediated interference) in S2-R+ cells. The MEGAscript ${ }^{T M}$ RNAi kit (ThermoFisher Scientific) was used to produce dsRNAs required for this experiment. Briefly, DNA templates containing promoter sequences on either 5' end were produced through PCR with T7-promoter-fused primers. $2 \mu \mathrm{g}$ of DNA template was transcribed in vitro for 4 hours as recommended by the manufacturer. The products were incubated at $75^{\circ} \mathrm{C}$ for 5 minutes and brought to room temperature to enhance dsRNA formation. A cocktail of DNAsel and RNAse removed DNA and ssRNAs, and the remaining dsRNA was purified using the provided reagents. All dsRNA reagents were verified by running on a $1 \%$ agarose gel and quantified by measuring absorbance at $260 \mathrm{~nm}$ using a NanoDrop ${ }^{\mathrm{TM}}$ (ThermoFisher Scientific).

For knockdown, $3 \times 10^{6} \mathrm{~S} 2-\mathrm{R}+$ cells in $1 \mathrm{~mL}$ serum free medium were incubated with 15 $\mu \mathrm{g}$ of dsRNA for 1 hour at room temperature. Then, $1 \mathrm{~mL}$ of medium containing $20 \%$ FBS was added to the cells and the whole mixture was moved to a 6 well plate. Cells were collected after 4 days of incubation.

\section{RT-PCR}

After transfection or RNAi treatment, cells were washed in ice cold PBS and pelleted using centrifugation. RNA was collected using the TRIzol reagent (Invitrogen) under the 
recommended conditions. $5 \mu \mathrm{g}$ of RNA was treated with Turbo DNase (Ambion) for 45 min before cDNA synthesis using SuperScript III (Life Technology) with random hexamers. RT-PCR was performed using AccuPrime Pfx DNA polymerase (ThermoFisher Scientific) with standard protocol using 26 cycles and primers that were specific to each minigene construct. All primers are listed and described in Supplementary Table 3.

\section{Acknowledgments}

Work in E.C.L.'s group was supported by the National Institutes of Health (R01NS083833 and R01-GM083300) and MSK Core Grant P30-CA008748.

Author contributions. BJJ designed the experiments, interpreted the data, and helped write the manuscript. ECL helped interpret data and write the manuscript. 


\section{References}

Akhtar J, Kreim N, Marini F, Mohana G, Brune D, Binder H, Roignant JY. 2019. Promoterproximal pausing mediated by the exon junction complex regulates splicing. Nature communications 10: 521.

Ashton-Beaucage D, Udell CM, Lavoie H, Baril C, Lefrancois M, Chagnon P, Gendron P, CaronLizotte O, Bonneil E, Thibault P et al. 2010. The exon junction complex controls the splicing of MAPK and other long intron-containing transcripts in Drosophila. Cell 143: 251-262.

Blazquez L, Emmett W, Faraway R, Pineda JMB, Bajew S, Gohr A, Haberman N, Sibley CR, Bradley RK, Irimia M et al. 2018. Exon Junction Complex Shapes the Transcriptome by Repressing Recursive Splicing. Molecular cell 72: 496-509 e499.

Boehm V, Britto-Borges T, Steckelberg AL, Singh KK, Gerbracht JV, Gueney E, Blazquez L, Altmuller J, Dieterich C, Gehring NH. 2018. Exon Junction Complexes Suppress Spurious Splice Sites to Safeguard Transcriptome Integrity. Molecular cell 72: 482-495 e487.

Boehm V, Gehring NH. 2016. Exon Junction Complexes: Supervising the Gene Expression Assembly Line. Trends in genetics : TIG 32: 724-735.

Bonnal SC, Lopez-Oreja I, Valcarcel J. 2020. Roles and mechanisms of alternative splicing in cancer - implications for care. Nat Rev Clin Oncol.

Brown JB, Boley N, Eisman R, May GE, Stoiber MH, Duff MO, Booth BW, Wen J, Park S, Suzuki AM et al. 2014. Diversity and dynamics of the Drosophila transcriptome. Nature 512: 393-399.

Burnette JM, Miyamoto-Sato E, Schaub MA, Conklin J, Lopez AJ. 2005. Subdivision of large introns in Drosophila by recursive splicing at nonexonic elements. Genetics 170: 661674.

Crooks GE, Hon G, Chandonia JM, Brenner SE. 2004. WebLogo: a sequence logo generator. Genome research 14: 1188-1190.

Drexler HL, Choquet K, Churchman LS. 2020. Splicing Kinetics and Coordination Revealed by Direct Nascent RNA Sequencing through Nanopores. Molecular cell 77: 985-998 e988.

Duff MO, Olson S, Wei X, Garrett SC, Osman A, Bolisetty M, Plocik A, Celniker SE, Graveley BR. 2015. Genome-wide identification of zero nucleotide recursive splicing in Drosophila. Nature 521: 376-379.

Hayashi R, Handler D, Ish-Horowicz D, Brennecke J. 2014. The exon junction complex is required for definition and excision of neighboring introns in Drosophila. Genes \& development 28: 1772-1785.

He F, Jacobson A. 2015. Nonsense-Mediated mRNA Decay: Degradation of Defective Transcripts Is Only Part of the Story. Annual review of genetics 49: 339-366.

Joseph B, Kondo S, Lai EC. 2018. Short cryptic exons mediate recursive splicing in Drosophila. Nature structural \& molecular biology 25: 365-371.

Kielkopf CL, Rodionova NA, Green MR, Burley SK. 2001. A novel peptide recognition mode revealed by the X-ray structure of a core U2AF35/U2AF65 heterodimer. Cell 106: 595605.

Kim D, Langmead B, Salzberg SL. 2015. HISAT: a fast spliced aligner with low memory requirements. Nature methods 12: 357-360.

Kim VN, Kataoka N, Dreyfuss G. 2001. Role of the nonsense-mediated decay factor hUpf3 in the splicing-dependent exon-exon junction complex. Science 293: 1832-1836.

Kondo Y, Oubridge C, van Roon AM, Nagai K. 2015. Crystal structure of human U1 snRNP, a small nuclear ribonucleoprotein particle, reveals the mechanism of 5 ' splice site recognition. elife 4. 
Le Hir H, Gatfield D, Izaurralde E, Moore MJ. 2001. The exon-exon junction complex provides a binding platform for factors involved in mRNA export and nonsense-mediated mRNA decay. The EMBO journal 20: 4987-4997.

Le Hir H, Izaurralde E, Maquat LE, Moore MJ. 2000. The spliceosome deposits multiple proteins 20-24 nucleotides upstream of mRNA exon-exon junctions. The EMBO journal 19: 68606869.

Le Hir H, Sauliere J, Wang Z. 2016. The exon junction complex as a node of post-transcriptional networks. Nature reviews Molecular cell biology 17: 41-54.

Lykke-Andersen J, Shu MD, Steitz JA. 2001. Communication of the position of exon-exon junctions to the mRNA surveillance machinery by the protein RNPS1. Science 293: 1836-1839.

Malone CD, Mestdagh C, Akhtar J, Kreim N, Deinhard P, Sachidanandam R, Treisman J, Roignant JY. 2014. The exon junction complex controls transposable element activity by ensuring faithful splicing of the piwi transcript. Genes \& development 28: 1786-1799.

Nicholson P, Muhlemann O. 2010. Cutting the nonsense: the degradation of PTC-containing mRNAs. Biochemical Society transactions 38: 1615-1620.

Palacios IM, Gatfield D, St Johnston D, Izaurralde E. 2004. An elF4Alll-containing complex required for mRNA localization and nonsense-mediated mRNA decay. Nature 427: 753757.

Reese MG, Eeckman FH, Kulp D, Haussler D. 1997. Improved splice site detection in Genie. Journal of computational biology : a journal of computational molecular cell biology 4: 311-323.

Robinson JT, Thorvaldsdottir H, Winckler W, Guttman M, Lander ES, Getz G, Mesirov JP. 2011. Integrative genomics viewer. Nature biotechnology 29: 24-26.

Roignant JY, Treisman JE. 2010. Exon junction complex subunits are required to splice Drosophila MAP kinase, a large heterochromatic gene. Cell 143: 238-250.

Sanfilippo P, Wen J, Lai EC. 2017. Landscape and evolution of tissue-specific alternative polyadenylation across Drosophila species. Genome biology 18: 229.

Schlautmann LP, Gehring NH. 2020. A Day in the Life of the Exon Junction Complex. Biomolecules 10.

Singh G, Kucukural A, Cenik C, Leszyk JD, Shaffer SA, Weng Z, Moore MJ. 2012. The cellular EJC interactome reveals higher-order mRNP structure and an EJC-SR protein nexus. Cell 151: 750-764.

Takahara K, Schwarze U, Imamura Y, Hoffman GG, Toriello H, Smith LT, Byers PH, Greenspan DS. 2002. Order of intron removal influences multiple splice outcomes, including a twoexon skip, in a COL5A1 acceptor-site mutation that results in abnormal pro-alpha1(V) Npropeptides and Ehlers-Danlos syndrome type I. Am J Hum Genet 71: 451-465.

Vaquero-Garcia J, Barrera A, Gazzara MR, Gonzalez-Vallinas J, Lahens NF, Hogenesch JB, Lynch KW, Barash Y. 2016. A new view of transcriptome complexity and regulation through the lens of local splicing variations. eLife 5: e11752.

Wei P, Xue W, Zhao Y, Ning G, Wang J. 2020. CRISPR-based modular assembly of a UAScDNA/ORF plasmid library for more than 5500 Drosophila genes conserved in humans. Genome research 30: 95-106.

Westholm JO, Miura P, Olson S, Shenker S, Joseph B, Sanfilippo P, Celniker SE, Graveley BR, Lai EC. 2014. Genome-wide Analysis of Drosophila Circular RNAs Reveals Their Structural and Sequence Properties and Age-Dependent Neural Accumulation. Cell reports 9: 1966-1980.

Wiegand HL, Lu S, Cullen BR. 2003. Exon junction complexes mediate the enhancing effect of splicing on mRNA expression. Proceedings of the National Academy of Sciences of the United States of America 100: 11327-11332. 
bioRxiv preprint doi: https://doi.org/10.1101/2020.07.31.231498; this version posted July 31, 2020. The copyright holder for this preprint (which

was not certified by peer review) is the author/funder, who has granted bioRxiv a license to display the preprint in perpetuity. It is made available under aCC-BY 4.0 International license.

Yu C, Wan KH, Hammonds AS, Stapleton M, Carlson JW, Celniker SE. 2011. Development of expression-ready constructs for generation of proteomic libraries. Methods in molecular biology 723: 257-272. 


\section{Figure Legends}

Figure 1. Transcriptome-wide de novo alternative splicing upon depletion of functional Exon Junction complex

(A) Overview of upregulated de novo splice junctions in EJC-depleted cells. Top: schematic of exonic and intronic cryptic 5' and 3' SS. Bottom: Pie chart indicating the distribution of different splice junction classes.

(B) Sashimi plot depicting HISAT2-mapped sequencing coverage along a portion of straw, which has defective splicing under core-EJC LOF. The gene model depicts the location of the cryptic 5' SS relative to the annotated 5' SS. Junction spanning read counts mapping to the canonical junction are circled, whereas cryptic junction read counts are squared. Note that spliced reads mapping to the cryptic junction are found in elF4AIII-, mago- and tsu-KD but not the control comparison. Region containing the cryptic 5'SS has been zoomed on the right. (C) Validation of de novo splicing events in core-EJC depleted cells. EJC core components (elF4AIII, mago, tsu and btz) were knocked down in Drosophila S2 cells using dsRNA. After knockdown, eight targets identified in (A) were evaluated using an rt-PCR assay and demonstrated splicing defects (asterisk). Importantly, only core-EJC factor KD produced cryptic bands, but not btz or control conditions. Note that several splicing defects are observed for unkempt (unk).

Figure 1 - figure supplement 1. core-EJC depletion yields broad activation of de novo splice junctions.

(A) Strong overlap of de novo splice junctions between core-EJC knockdown conditions based. The Venn diagram depicts which of 1677 junctions with at least 5 split reads had $>2$-fold split read changes between treatment and controls.p-value for three-way overlap was calculated using a permutation test with $10^{8}$ tests. 
(B) Strong overlap of high-confidence de novo splice junctions between core-EJC knockdown conditions. The Venn diagram depicts which of 876 junctions with at least 5 split reads and > 2fold split read changes also show > 2-fold changes in percent selected index (PSI) between treatment and controls. p-value for three-way overlap was calculated using a permutation test with $10^{8}$ tests.

(C) Knockdown of EJC factors in S2 cells using dsRNA. quantitative rt-PCR of core-EJC and btz transcripts after dsRNA treatment.

\section{Figure 2. EJC-depletion leads to activation of cryptic 3' splice sites}

(A) Depiction of 3' SS position of spurious junctions relative to exon-exon boundaries as density and dot plot. The dot plot indicates splice site scores as calculated via NNSPLICE. Horizontal dashed line depicts threshold for strong 3' SS, and vertical dashed lines specify 50 nt flanking exon-exon junctions.

(B) Sashimi plot depicting HISAT2-mapped sequencing coverage along a portion of CG7408, which has a cryptic 3' SS that is activated under core-EJC LOF. Junction spanning read counts mapping to the canonical junction are circled, whereas cryptic junction read counts are squared. Note that spliced reads mapping to the cryptic junction are found in elF4AIII, mago and tsu KD but not the control comparison.

(C) Validation of CG7408 cryptic 3' SS activation in core-EJC, but not btz or lacZ KD conditions

(D) Schematic of CG7408 splicing reporters. Exons 1-4 (introns included) were cloned and subjected to further manipulation. Locations of pre-removed introns $(\Delta)$, as well as a construct lacking all introns (mRNA) are included. For reference, the position of the cryptic 3'SS is marked on exon 2. genomic+spacer represents a modified version of the genomic splicing reporter with an insertion of $36 \mathrm{nt}$ spacer sequence on exon 2. 
(E) rt-PCR of reporter (D) constructs ectopically expressed in S2 cells demonstrates that intron 2 is required for accurate processing of the minigene. Canonical and cryptic products are indicated.

(F) Cryptic splicing is detected with the inclusion of a $36 \mathrm{nt}$ spacer sequence.

(G) Schematic of out-of-order splicing and positional requirement of the core-EJC for accurate 3' SS definition.

Figure 2 - figure supplement 1. A majority of cryptic 3' SS activated under EJC-loss are weak

(A) Nucleotide content of cryptic 3' SS. These sequences, apart from the invariant AG dinucleotide show poor strength.

(B) Example of a weak cryptic 3' SS (NNSPLICE score of 0.29 ) found on the CG7408 transcript. Conservation of the weak splice site is depicted using the multiple alignment format on the UCSC genome browser, as well as phyloP and phastCons scores.

\section{Figure 3. EJC-depletion leads to activation of cryptic 5' splice sites}

(A) Metagene of cryptic 5' SS position relative to exon-exon boundaries as density and dot plot. The dot plot indicates splice site scores as calculated via NNSPLICE (see Methods). Horizontal dashed line depicts threshold for strong 3' SS, and vertical dashed lines specify 50 nt flanking exon-exon junctions.

(B) Sashimi plot depicting HISAT2-mapped sequencing coverage along a portion of CG3632, which has a cryptic 5' SS that is activated under core-EJC LOF. Junction spanning read counts mapping to the canonical junction are circled, whereas cryptic junction read counts are squared. Note that spliced reads mapping to the cryptic junction are found in elF4AIII, mago and tsu KD but not the control comparison. 
(C) Validation of CG3632 cryptic 5' SS activation (asterisk) in core-EJC, but not btz or lacZ KD conditions

(D) Schematic of CG3632 splicing reporters. Exons 13-15 (introns included) were cloned and subjected to further manipulation. Locations of pre-removed introns $(\Delta)$, as well as a construct lacking all introns (mRNA) are included. The position of the cryptic 5'SS is marked on exon 14, and was mutated in $\triangle \mathrm{i} 13+\mathrm{SD}$ mut.

(E) rt-PCR of reporter (D) constructs ectopically expressed in S2 cells demonstrates that intron 13 is required for accurate processing of the minigene. Canonical products are indicated by the line and cryptic products by an asterisk.

Figure 3 - figure supplement 1. A majority of cryptic 5' SS activated under EJC-loss are weak

(A) Nucleotide content of cryptic 5' SS.

(B) Schematic of a de novo splicing event detected on the CG3632 transcript. Validation of splicing defects shown on the right.

(C) Cryptic 5' SS (NNSPLICE score of 0.54 ) found on the CG3632 transcript. Conservation of the weak splice site is depicted using the multiple alignment format on the UCSC genome browser, as well as phyloP and phastCons scores.

Figure 4. EJC-depletion leads to activation of dual cryptic splice sites and resplicing of mRNA

(A) Above: Schematic of resplicing splicing versus alternative resplicing, both of which would yield the same aberrant mRNA product. Below: Sequence of $C k \| \beta$ transcript lost due to cryptic splicing. Cryptic 3' SS activated is highlighted in red, as well as a potential regenerated 5' SS. Scores listed are generated by NNSPLICE. Conservation across Drosophilid family is shown. 
(B) Schematic of Ckllß splicing reporters. Exons 2-4 (introns included) were cloned and subjected to further manipulation. Locations of pre-removed introns $(\Delta)$, as well as a construct lacking all introns (mRNA) are included. For reference, the position of the cryptic 3' SS and potential $5^{\prime}$ recursive splice sites is marked on exon 3.

(C) rt-PCR of $C k l l \beta$ reporter constructs in S2 cells demonstrates that introns are required for accurate processing of the minigene. Canonical and cryptic products are indicated.

(D) Validation of CG31156 cryptic 5' SS activation in core-EJC, but not btz or lacZ KD conditions (E) Schematic of CG31156 splicing reporters with and without introns. Location of potential 3' recursive splice site on exon 2 is indicated along with conservation scores.

(F) rt-PCR of reporter constructs in S2 cells demonstrates that introns are required for accurate processing of the minigene. Canonical and cryptic products are indicated.

(G) Model for mRNA resplicing. Top, Binding sites of U1 snRNA and U2AF35 define the 5' SS and 3 ' SS, respectively, but also impose constraints on flanking exonic sequences that intrinsically regenerate splice site mimics in a recursive fashion. (Bottom) When located in proximity to another cryptic splice site, these can lead to mRNA resplicing in the absence of the EJC. An example of dual cryptic splice sites with a regenerated 3' SS is shown, but this can also occur with a regenerated 5' SS.

(H) Comparison of splice site strengths for cases of dual cryptic splice site activation. Cases that contain regenerated $3^{\prime}$ and $5^{\prime}$ splice sites at exon junctions and their structures are schematized and distinguished by red and blue dot. Dashed lines mark thresholds for reasonably strong splice sites.

(I) Resplicing on cDNAs. Constructs bearing cDNA segments of baboon, elF4G1 and straw were expressed in S2 cells and yielded re-spliced amplicons. Gene specific primers that amplify endogenous and ectopic products only show resplicing from the intron-less reporter. Transgenespecific primers demonstrate mostly re-spliced products. 
Figure 4 - figure supplement 1. de novo splicing on CkIIß is a result of dual cryptic splice site activation.

(A) Sashimi plot depicting HISAT2-mapped sequencing coverage along a portion of CkIIß, which has a cryptic 3' SS that is activated under core-EJC LOF. Junction spanning read counts mapping to the canonical junction are circled, whereas cryptic junction read counts are squared. Note that spliced reads mapping to the cryptic junction are found in eIF4AIII, mago and tsu but not the control comparison.

(B) Schematic of a de novo splicing event detected on the CkIIß transcript.

(C) Validation of CkIIß cryptic 3' SS activation in core-EJC, but not btz or lacZ KD conditions

(D) Models that explain the CkIIß splicing defects. Path 1 and 2 reflect alternate orders of intron removal. Crucially, path 1 leads to EJC-suppressed cryptic splicing on mRNAs using the indicated 5' recursive splice site and a cryptic 3'SS, whereas path 2 can also produce a splice defect after removal of intron 2.

Figure 4 - figure supplement 2. de novo splicing on CG31156 is a result of dual cryptic splice site activation.

(A) Sashimi plot depicting HISAT2-mapped sequencing coverage along a portion of CG31156, which has a cryptic 5' SS that is activated under core-EJC LOF. Junction spanning read counts mapping to the canonical junction are circled, whereas cryptic junction read counts are squared. Note that spliced reads mapping to the cryptic junction are found in eIF4AIII, mago and tsu but not the control comparison.

(B) Schematic of a de novo splicing event detected on the CG31156 transcript.

(C) Conservation of the cryptic 5' SS (NNSPLICE score of 0.54 ) and a potential 3' recursive splice site (NNSPLICE score of 0.98) found on the CG31156 transcript highlighted in green, relative to the gene model. Conservation of the splice site is depicted using the multiple 
alignment format on the UCSC genome browser, as well as phyloP scores. Canonical splice sites are highlighted in yellow.

(D) Model of activation of dual cryptic splice sites on the CG31156 transcript. Activation of the cryptic 5' SS with an additional cryptic 3' recursive splice sites leads to deletion of $110 \mathrm{nt}$ of mRNA.

\section{Figure 4 - figure supplement 3. Resplicing on mRNAs alters translated proteins}

(A-C) Protein and transcript structures are schematized and the location of cryptic resplicing highlighted in blue.

(A) Resplicing on baboon leads to a 54 nt deletion of the mRNA and an 18 amino acid deletion. The deletion does not overlap known domains. Conservation plots for deleted $54 \mathrm{nt}$ region is included.

(B) Resplicing on elF4G1 leads to a $131 \mathrm{nt}$ deletion, leading to a change in reading frame and truncation of the $\mathrm{C}$ terminal domains of elF4G1. Importantly, critical domains required for elF4G1 function are lost due to resplicing.

(C) Resplicing on straw leads to a $91 \mathrm{nt}$ deletion, leading to a change in reading frame and truncation of the protein. Importantly, 2 of 3 Plastocyanin-like domains are lost due to transcript defects. 

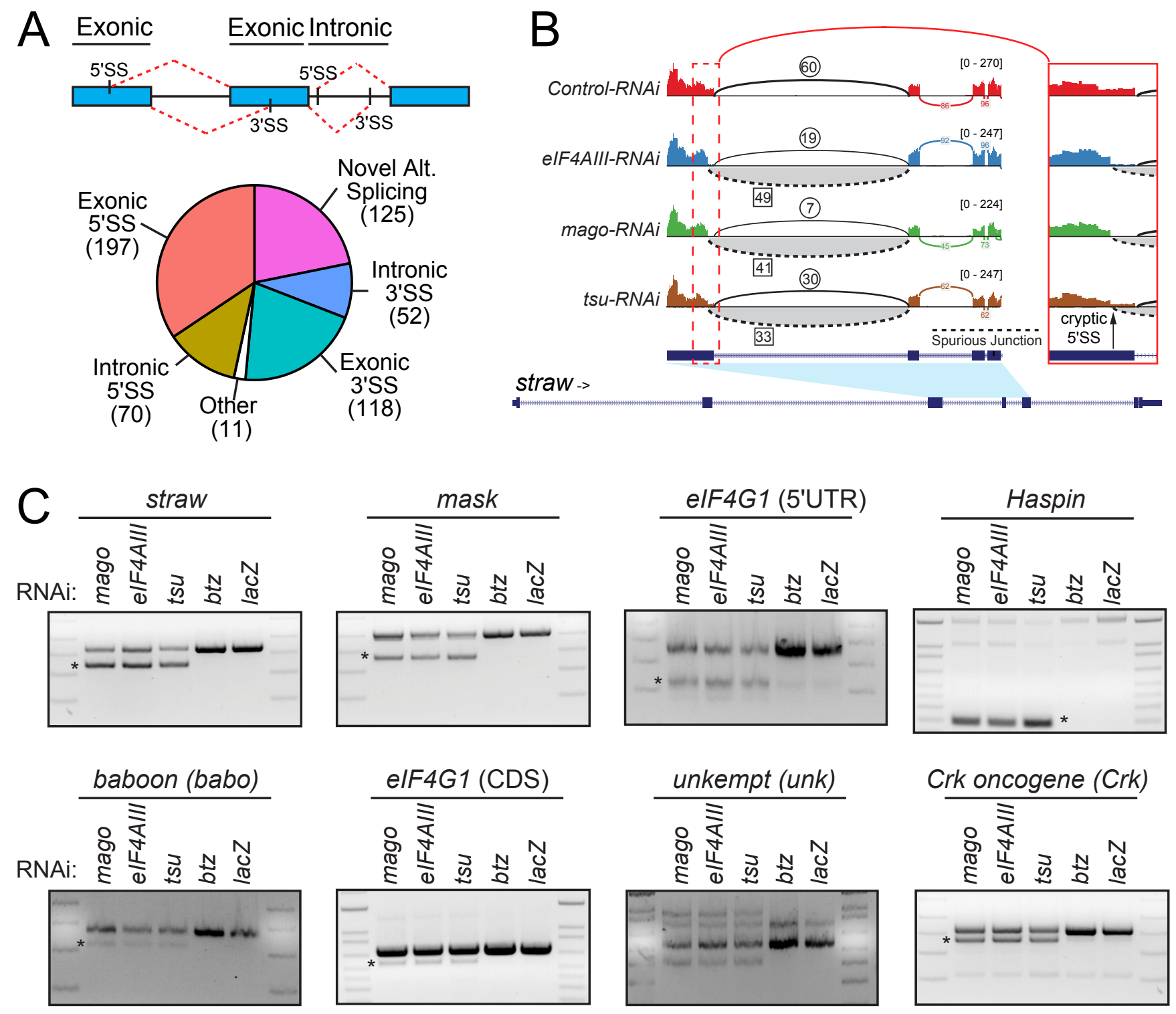

Figure 1. Transcriptome-wide alternative splicing upon depletion of functional Exon Junction complex

(A) Overview of upregulated de novo splice junctions in EJC-depleted cells. Top: schematic of exonic and intronic cryptic 5' and 3' SS. Bottom: Pie chart indicating the distribution of different splice junction classes.

(B) Sashimi plot depicting HISAT2-mapped sequencing coverage along a portion of straw, which has defective splicing under core-EJC LOF. The gene model depicts the location of the cryptic 5' SS relative to the annotated 5' SS. Junction spanning read counts mapping to the canonical junction are circled, whereas cryptic junction read counts are squared. Note that spliced reads mapping to the cryptic junction are found in elF4AIII-, mago- and tsu-KD but not the control comparison.

(C) Validation of de novo splicing events in core-EJC depleted cells. EJC core components (elF4AIII, mago, tsu and btz) were knocked down in Drosophila S2 cells using dsRNA. After knockdown, eight targets identified in (A) were evaluated using an rt-PCR assay and demonstrated splicing defects (asterisk). Importantly, only core-EJC factor KD produced cryptic bands, but not btz or control conditions. 
bioRxiv preprint doi: https://doi.org/10.1101/2020.07.31.231498; this version posted July 31, 2020. The copyright holder for this preprint (which

was not certified by peer review) is the author/funder, who has granted bioRxiv a license to display the preprint in perpetuity. It is made available under aCC-BY 4.0 International license.

Overlap of de novo junctions

A with $>5$ split reads $+>2$ split read FC

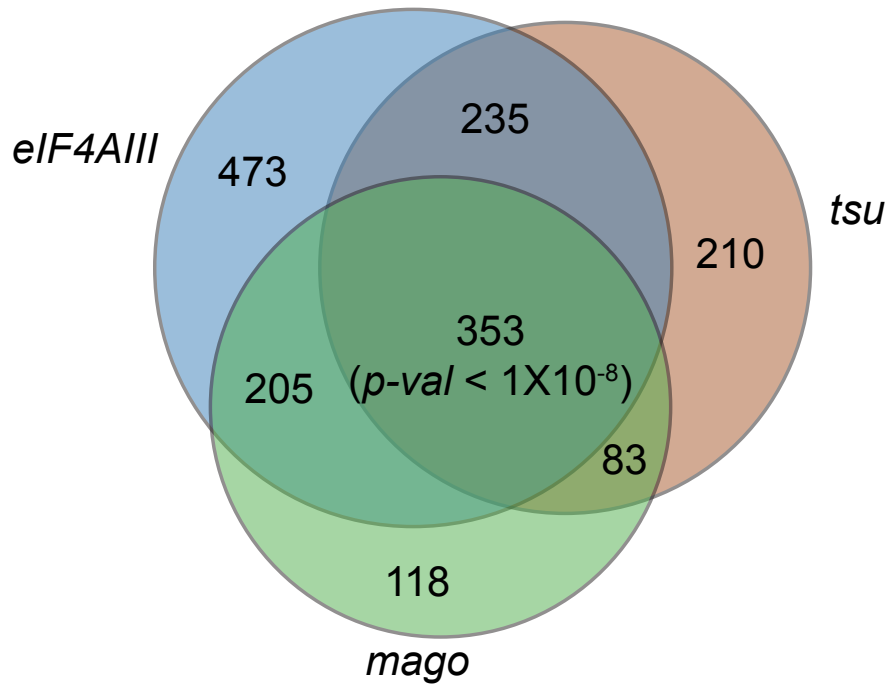

Overlap of de novo junctions

B with $>5$ split reads $+>2$ split read FC $+>2$ PSI FC

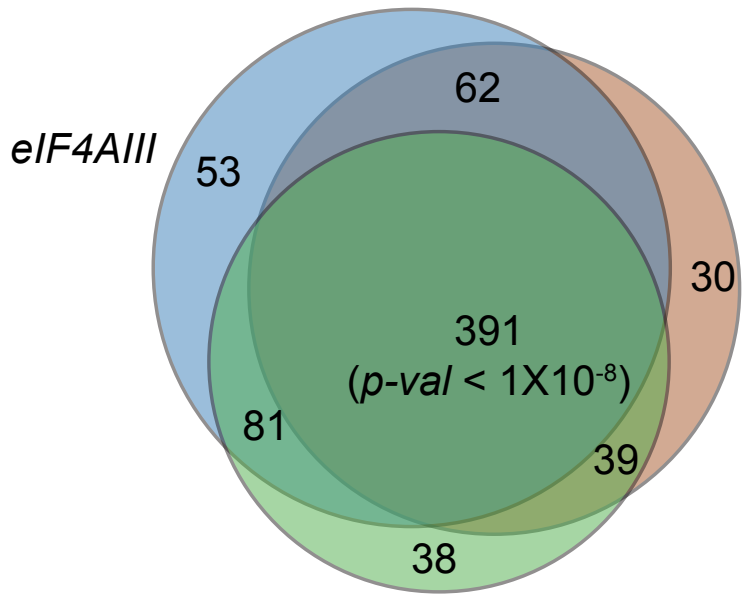

mago

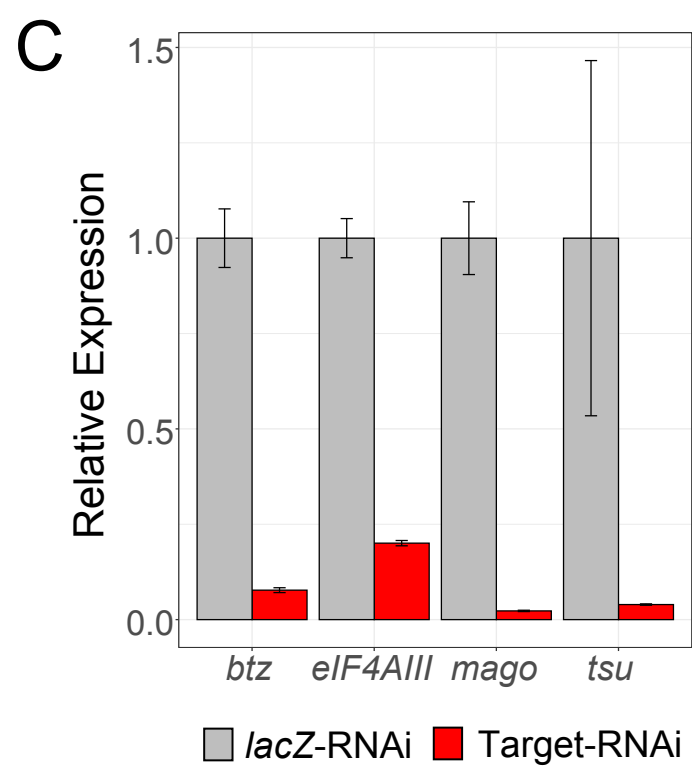

Figure 1 - figure supplement 1. core-EJC depletion yields broad activation of de novo splice junctions.

(A) Strong overlap of de novo splice junctions between core-EJC knockdown conditions based. The Venn diagram depicts which of 1677 junctions with at least 5 split reads had $>2$-fold split read changes between treatment and controls.p-value for three-way overlap was calculated using a permutation test with $10^{8}$ tests.

(B) Strong overlap of high-confidence de novo splice junctions between core-EJC knockdown conditions. The Venn diagram depicts which of 876 junctions with at least 5 split reads and $>2$-fold split read changes also show $>2$-fold changes in percent selected index (PSI) between treatment and controls. $p$-value for three-way overlap was calculated using a permutation test with $10^{8}$ tests.

(C) Knockdown of EJC factors in S2 cells using dsRNA. quantitative rt-PCR of core-EJC and btz transcripts after dsRNA treatment. 
A Cryptic 3 ' splice site strength

$A$ and exon-exon junction position

\section{Joseph_Fig2}

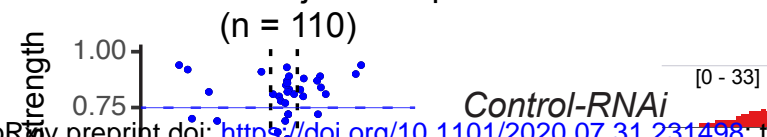

bioR

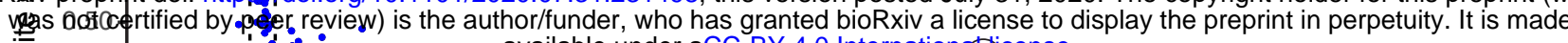

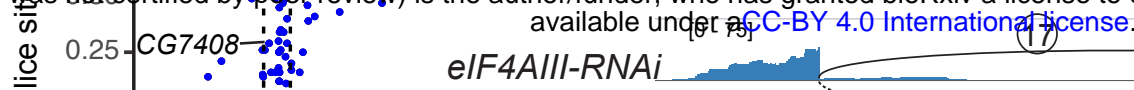
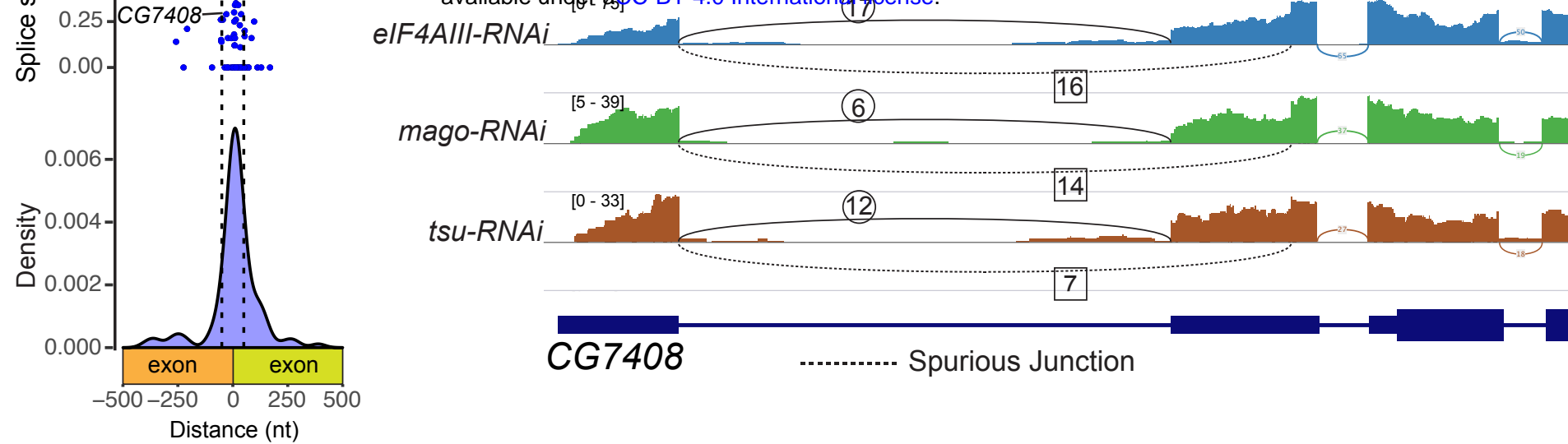

tsu-RNA

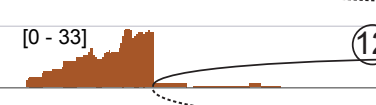

14

7
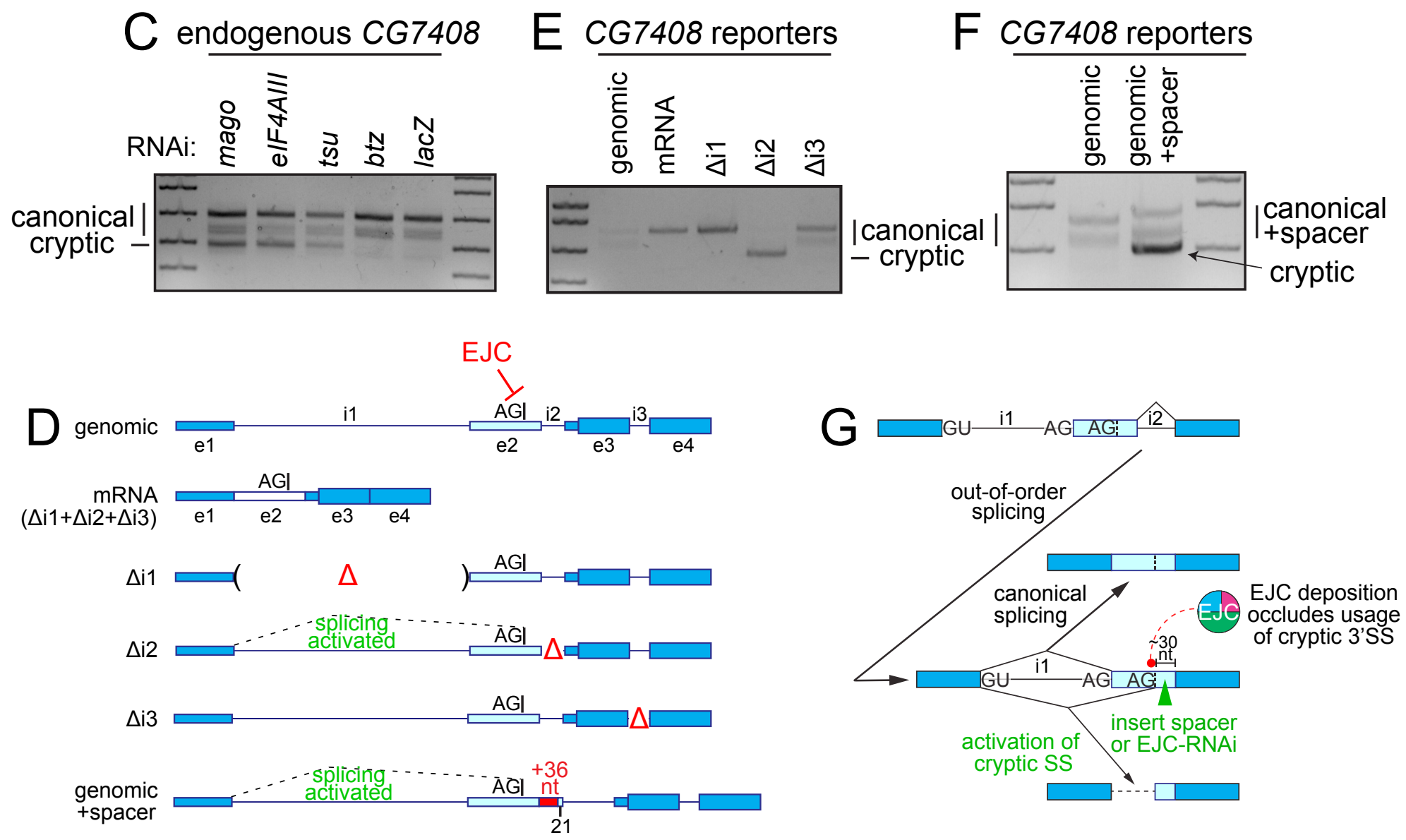

Figure 2. EJC-depletion leads to activation of cryptic 3' splice sites

(A) Depiction of 3' SS position of spurious junctions relative to exon-exon boundaries as density and dot plot. The dot plot indicates splice site scores as calculated via NNSPLICE. Horizontal solid line depicts a threshold for strong 3' SS.

(B) Sashimi plot depicting HISAT2-mapped sequencing coverage along a portion of CG7408, which has a cryptic 3' SS that is activated under core-EJC LOF. Junction spanning read counts mapping to the canonical junction are circled, whereas cryptic junction read counts are squared. Note that spliced reads mapping to the cryptic junction are found in elF4Alll, mago and tsu KD but not the control comparison.

(C) Validation of CG7408 cryptic 3' SS activation in core-EJC, but not btz or lacZ KD conditions

(D) Schematic of CG7408 splicing reporters. Exons 1-4 (introns included) were cloned and subjected to further manipulation. Locations of pre-removed introns $(\Delta)$, as well as a construct lacking all introns (mRNA) are included. For reference, the position of the cryptic 3'SS is marked on exon 2. The bottom construct contains insertion of a $36 \mathrm{nt}$ spacer sequence.

(E) rt-PCR of reporter (D) constructs ectopically expressed in S2 cells demonstrates that intron 2 is required for accurate processing of the minigene. Canonical and cryptic products are indicated.

(F) Cryptic splicing is detected upon inclusion of a $36 \mathrm{nt}$ spacer sequence.

(G) Schematic of out-of-order splicing and positional requirement of the core-EJC for accurate 3' SS definition. 

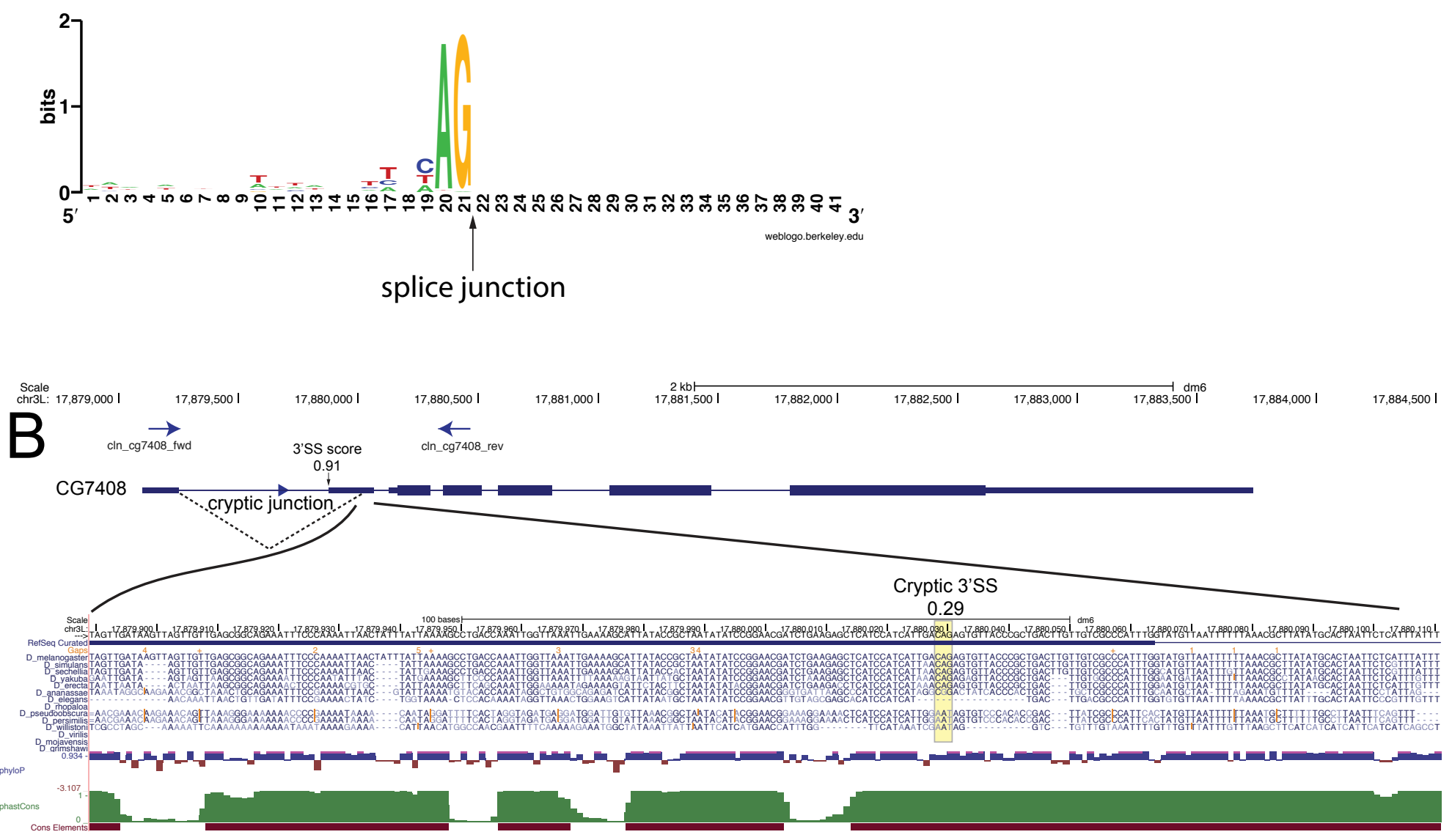

Figure 2 - figure supplement 1. A majority of cryptic 3' SS activated under EJC-loss are weak (A) Nucleotide content of cryptic 3' SS. These sequences, apart from the invariant AG dinucleotide show poor strength.

(B) Example of a weak cryptic 3' SS (NNSPLICE score of 0.29 ) found on the CG7408 transcript. Conservation of the weak splice site is depicted using the multiple alignment format on the UCSC genome browser, as well as phyloP and phastCons scores. 


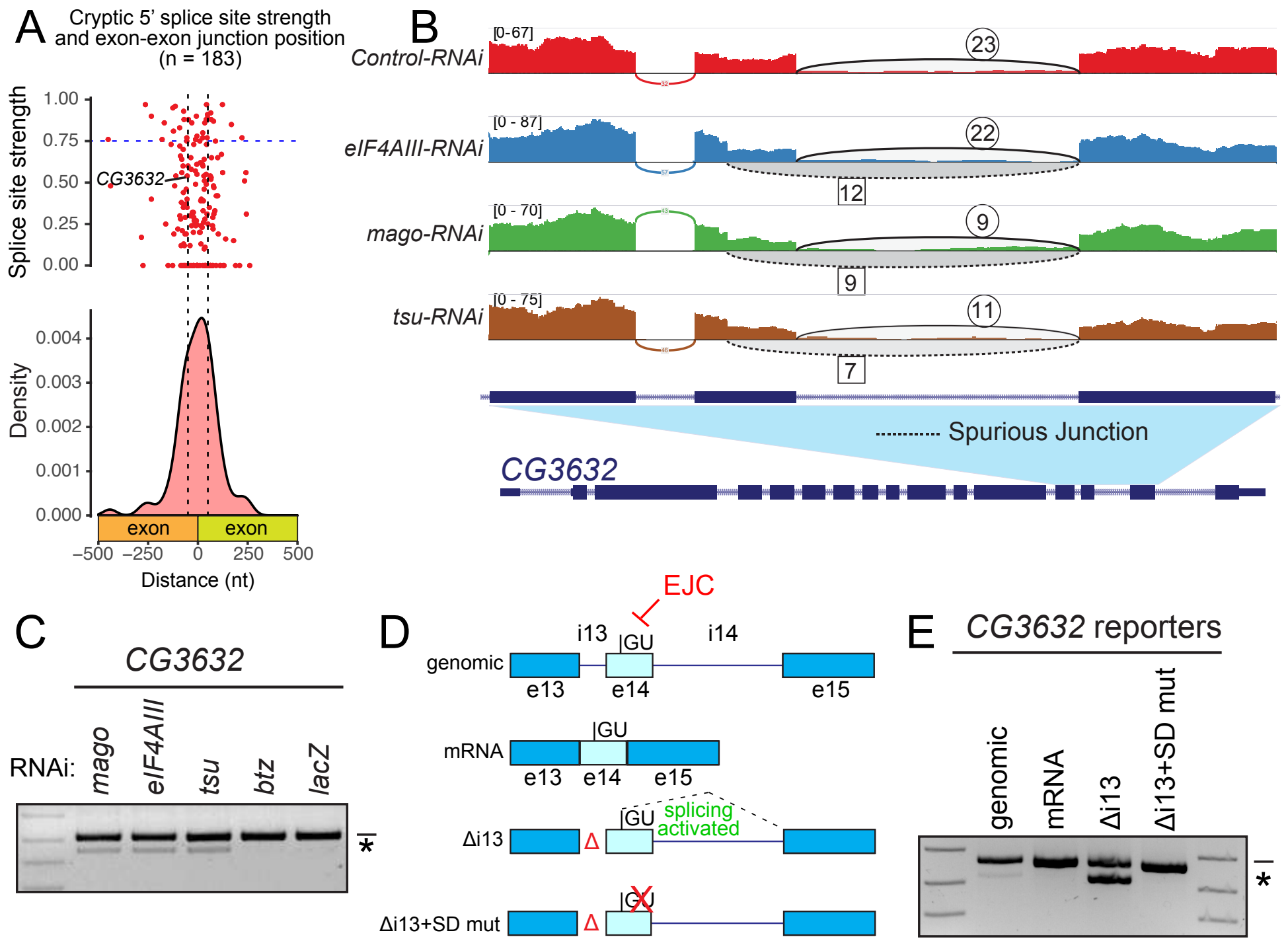

Figure 3. EJC-depletion leads to activation of cryptic 5' splice sites.

(A) Metagene of cryptic 5' SS position relative to exon-exon boundaries as density and dot plot. The dot plot indicates splice site scores as calculated via NNSPLICE (see Methods). Horizontal solid line depicts a threshold for strong 5' SS. (B) Sashimi plot depicting HISAT2-mapped sequencing coverage along a portion of CG3632, which has a cryptic 5' SS that is activated under core-EJC LOF. Junction spanning read counts mapping to the canonical junction are circled, whereas cryptic junction read counts are squared. Note that spliced reads mapping to the cryptic junction are found in elF4AIII, mago and tsu KD but not the control comparison.

(C) Validation of CG3632 cryptic 5' SS activation (asterisk) in core-EJC, but not btz or lacZ KD conditions.

(D) Schematic of CG3632 splicing reporters. Exons 13-15 (introns included) were cloned and subjected to further manipulation. Locations of pre-removed introns $(\Delta)$, as well as a construct lacking all introns (mRNA) are included. The position of the cryptic 5'SS is marked on exon 14, and was mutated in $\triangle \mathrm{i} 3+\mathrm{SD}$ mut.

(E) rt-PCR of reporter (D) constructs expressed in S2 cells demonstrates that intron 13 is required for accurate processing of the minigene. Canonical products are indicated by the line and cryptic products by an asterisk. 


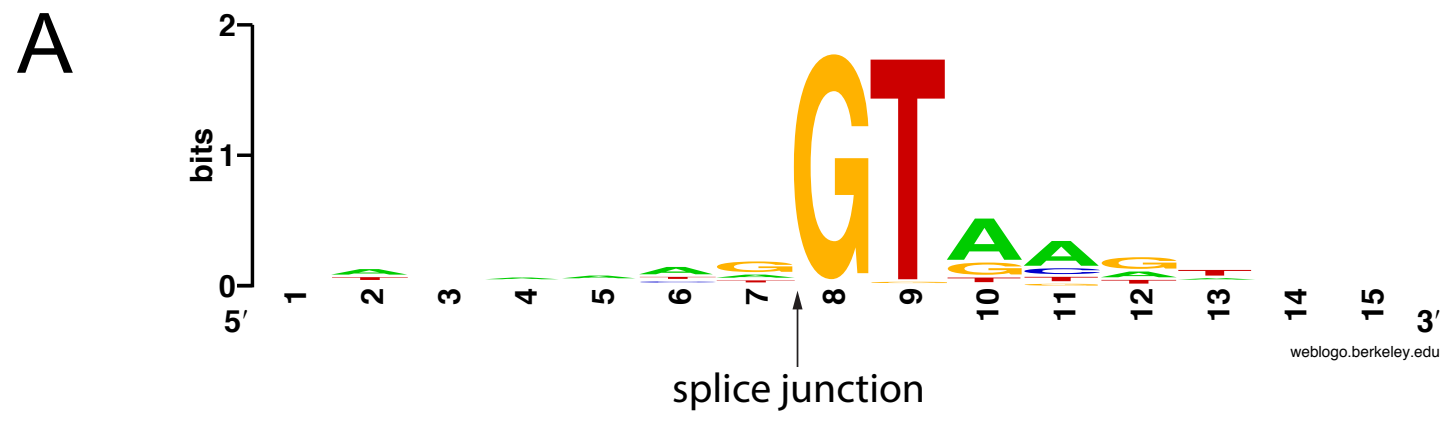

B
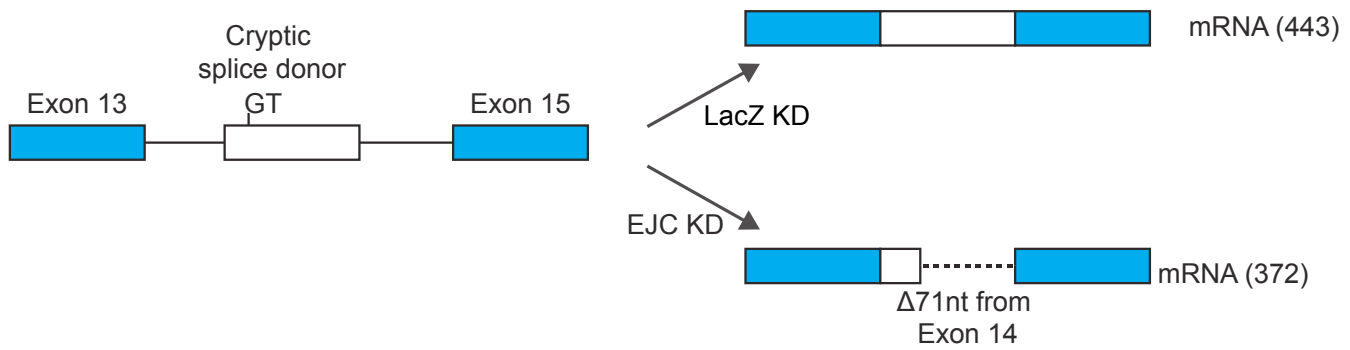

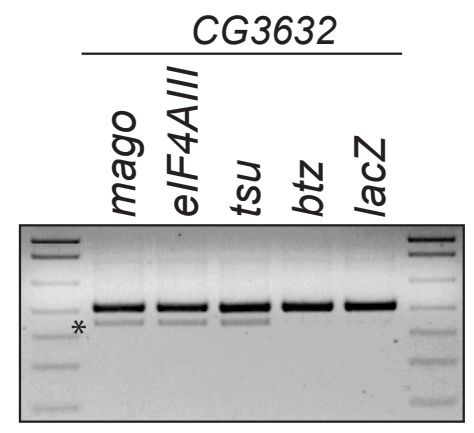

C Cryptic 5'ss

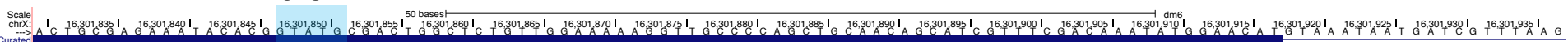

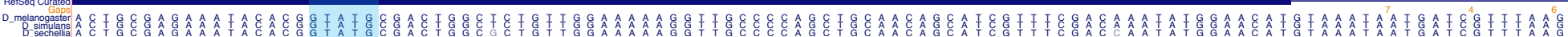

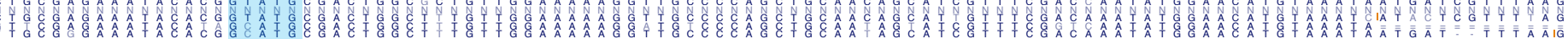

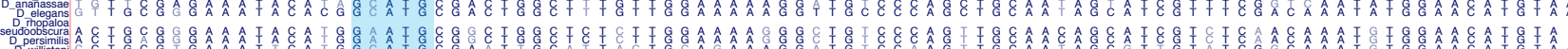

Figure 3 - figure supplement 1. A majority of cryptic 5' SS activated under EJC-loss are weak

(A) Nucleotide content of cryptic 5' SS.

(B) Schematic of a de novo splicing event detected on the CG3632 transcript. Validation of splicing defects shown on the right.

(C) Cryptic 5' SS (NNSPLICE score of 0.54) found on the CG3632 transcript. Conservation of the weak splice site is depicted using the multiple alignment format on the UCSC genome browser, as well as phyloP and phastCons scores. 


\section{Joseph_Fig4}
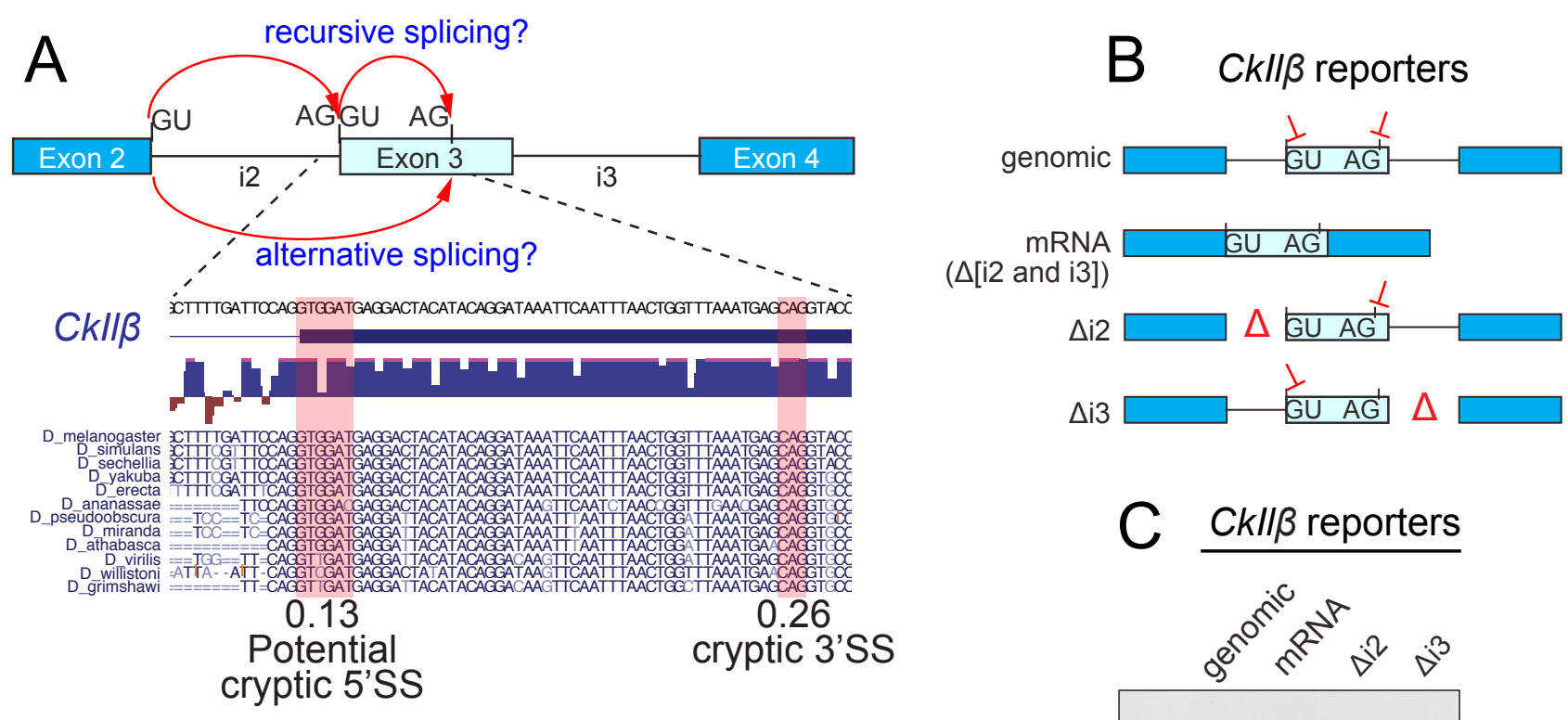

\section{Ckllß reporters}

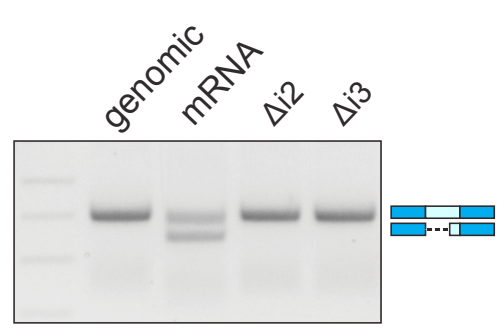

D $\frac{\text { CG31156 }}{\mathrm{E}} \begin{gathered}\text { Potential } \\ \text { cryptic 3'SS } \\ 0.98\end{gathered}$

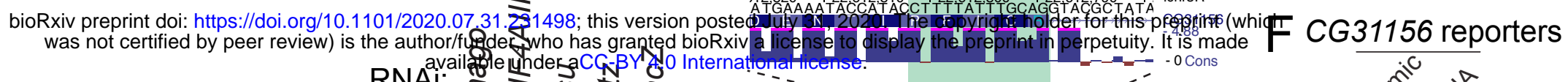
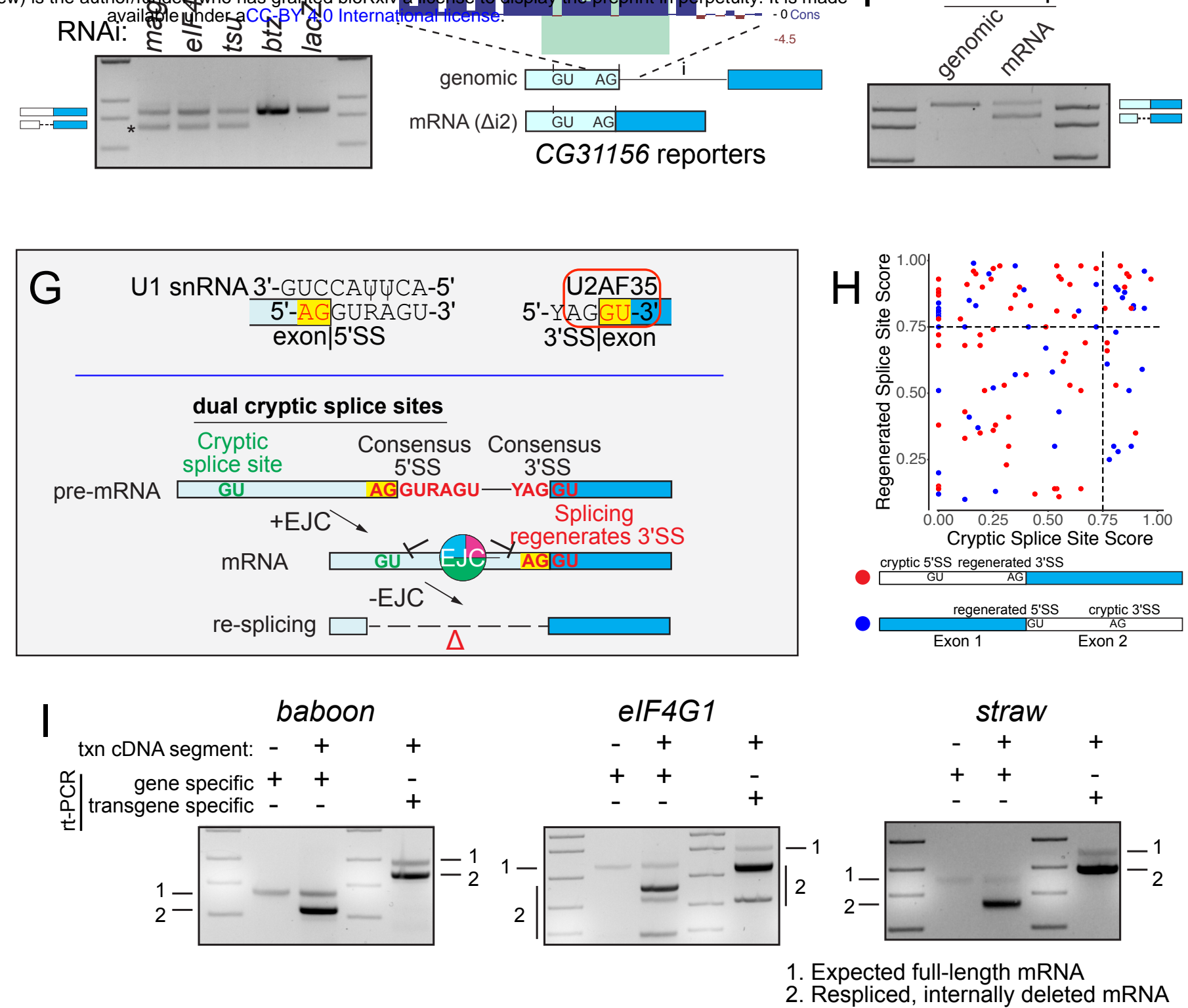

Figure 4. EJC-depletion leads to activation of dual cryptic splice sites and resplicing on mRNAs.

(A) Above: Schematic of resplicing splicing versus alternative resplicing, both of which would yield the same aberrant mRNA product. Below: Sequence of CkIl $\beta$ transcript lost due to cryptic splicing. Cryptic 3' SS activated is highlighted, as well as potential regenerated 5' SS. Scores listed are generated by NNSPLICE. Conservation across Drosophilid species is shown.

(B) Schematic of $C k l l \beta$ splicing reporters. Exons 2-4 (introns included) were cloned and subjected to further manipulation. Locations of pre-removed introns $(\Delta)$, as well as a construct lacking all introns (mRNA) are included. For reference, the position of the cryptic 3' SS and potential 5' recursive splice sites is marked on exon 3.

(C) rt-PCR of $C k \| l \beta$ reporter constructs in S2 cells demonstrates that introns are required for accurate processing of the minigene. Canonical and cryptic products are indicated.

(D) Validation of CG31156 cryptic 5' SS activation in core-EJC, but not btz or lacZ KD conditions

(E) Schematic of CG31156 splicing reporters with and without introns. Location of potential 3' recursive splice site on exon 2 along with conservation.

(F) rt-PCR of reporter constructs in S2 cells demonstrates that introns are required for accurate processing of the minigene. Canonical and cryptic products are indicated.

(G) Model for mRNA re-splicing. Top, Binding sites of U1 snRNA and U2AF35 define the 5'SS and 3'SS, respectively, but also impose constraints on flanking exonic sequences that intrinsically regenerate splice site mimics in a recursive fashion. (Bottom) When located in proximity to another cryptic splice site, these can lead to mRNA resplicing in the absence of the EJC. An example of dual cryptic splice sites with a regenerated 3'SS is shown, but this can also occur with a regenerated 5' SS.

(H) Comparison of splice site strengths for cases of dual cryptic splice site activation. Cases that contain regenerated 3' and 5' splice sites at exon junctions and their structures are schematized and distinguished by red and blue dot. Dashed lines mark thresholds for reasonably strong splice sites. (I) Re-splicing on cDNAs. Constructs bearing cDNA segments of baboon, elF4G1 and straw were expressed in S2 cells and yielded re-spliced amplicons. Gene specific primers that amplify endogenous and ectopic products only show re-splicing from the intron-less reporter. Transgene-specific primers demonstrate mostly respliced products. 
bioRxiv preprint doi: https://doi.org/10.1101/2020.07.31.231498; this version posted July 31, 2020. The copyright holder for this preprint (which was not certified by peer review) is the author/funder, who has granted bioRxiv a license to display the preprint in perpetuity. It is made
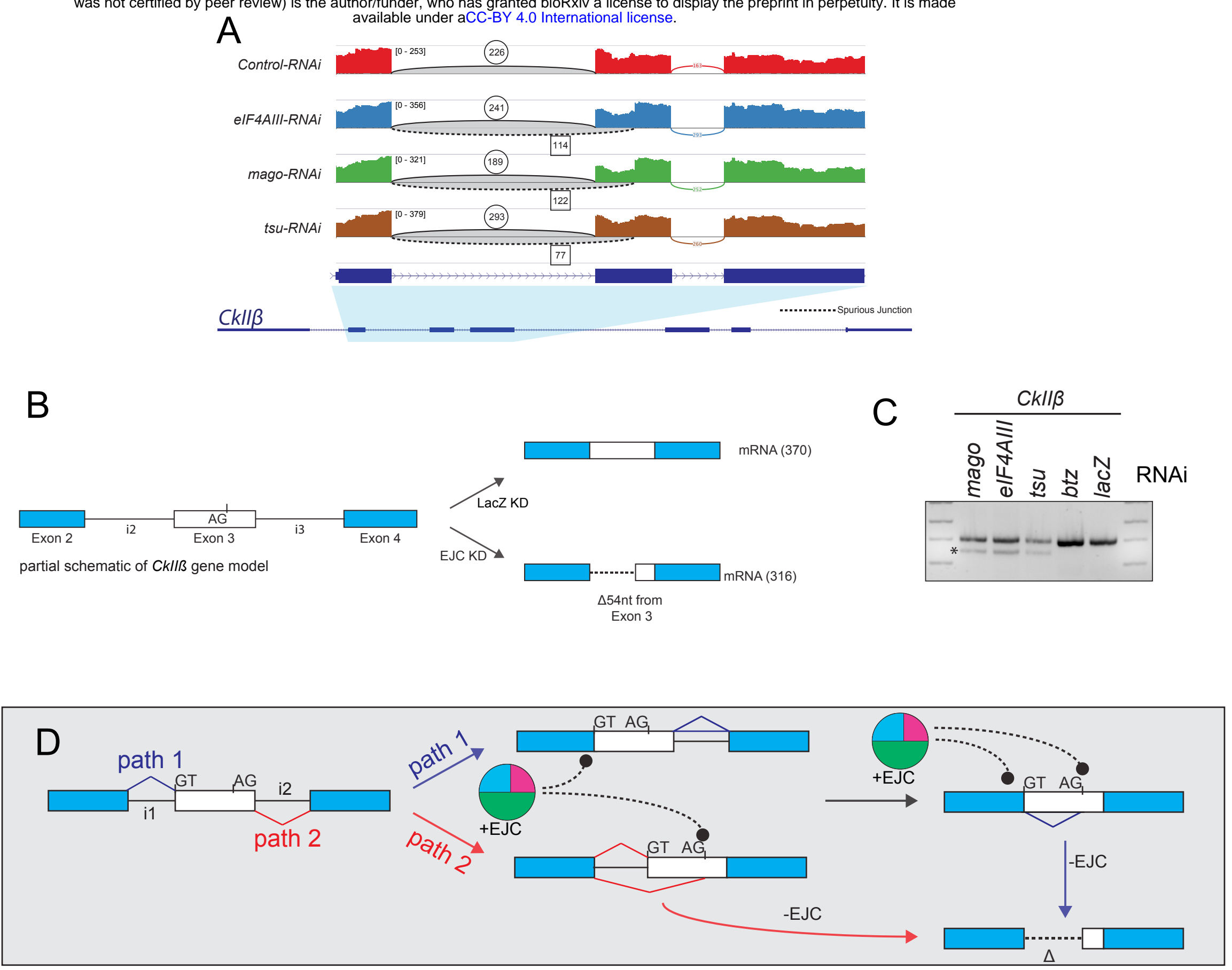

Figure 4 - figure supplement 1 . de novo splicing on CkIIß is a result of dual cryptic splice site activation.

(A) Sashimi plot depicting HISAT2-mapped sequencing coverage along a portion of CkIIß, which has a cryptic 3' SS that is activated under core-EJC LOF. Junction spanning read counts mapping to the canonical junction are circled, whereas cryptic junction read counts are squared. Note that spliced reads mapping to the cryptic junction are found in elF4AIII, mago and tsu but not the control comparison.

(B) Schematic of a de novo splicing event detected on the CkIIß transcript.

(C) Validation of CkIIß cryptic 3' SS activation in core-EJC, but not btz or lacZ KD conditions

(D) Models that explain the CkIIß splicing defects. Path 1 and 2 reflect alternate orders of intron removal. Crucially, path 1 leads to EJC-suppressed cryptic splicing on mRNAs using the indicated 5' recursive splice site and a cryptic 3'SS, whereas path 2 can also produce a splice defect after removal of intron 2. 
bioRxiv preprint doi: https://doi.org/10.1101/2020.07.31.231498; this version posted July 31, 2020. The copyright holder for this preprint (which was not certified by peer review) is the author/funder, who has granted bioRxiv a license to display the preprint in perpetuity. It is made available under aCC-BY 4.0 International license.
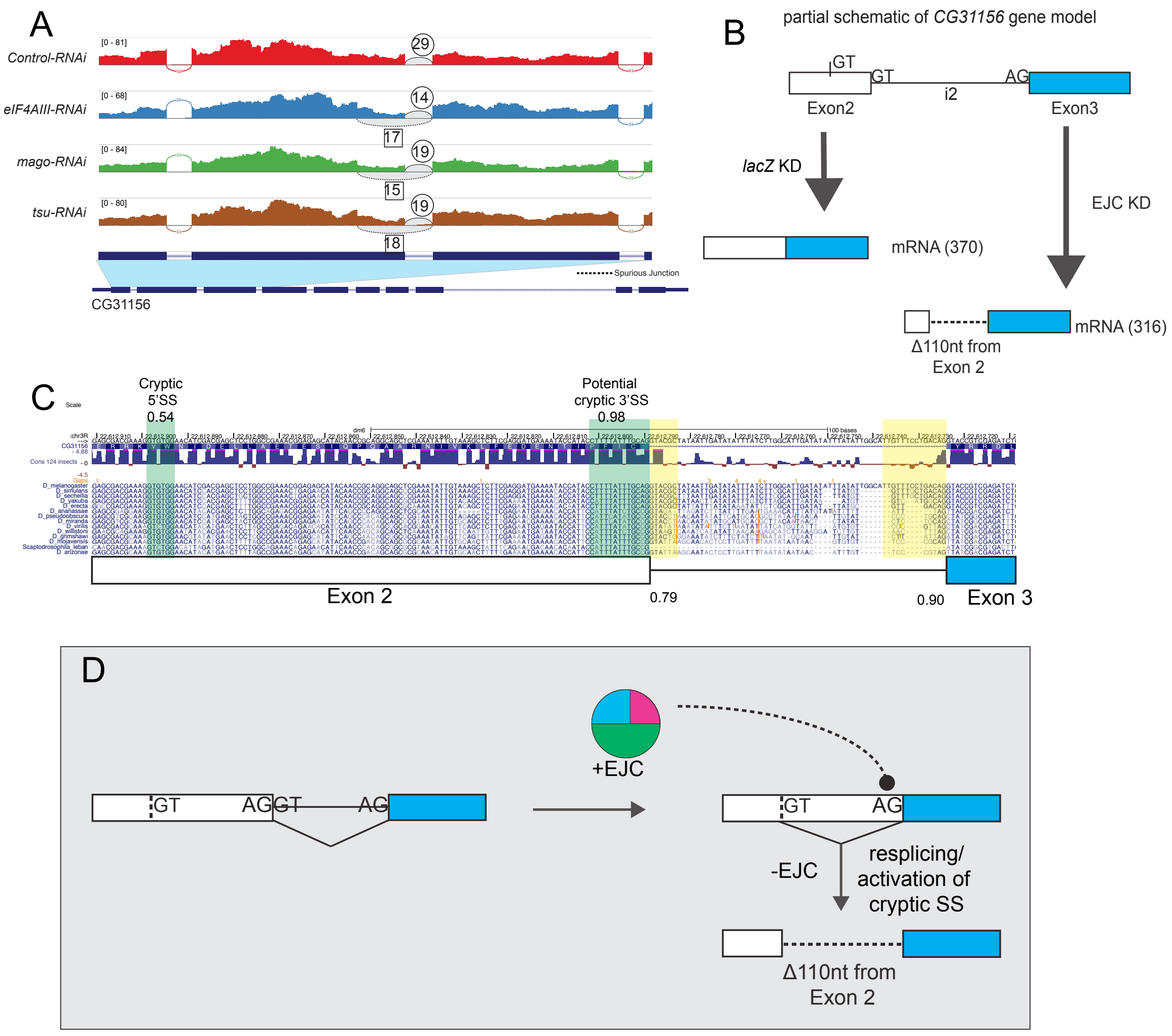

Figure 4 - figure supplement 2. de novo splicing on CG31156 is a result of dual cryptic splice site activation.

(A) Sashimi plot depicting HISAT2-mapped sequencing coverage along a portion of CG31156, which has a cryptic 5' SS that is activated under core-EJC LOF. Junction spanning read counts mapping to the canonical junction are circled, whereas cryptic junction read counts are squared. Note that spliced reads mapping to the cryptic junction are found in elF4AIII, mago and tsu but not the control comparison.

(B) Schematic of a de novo splicing event detected on the CG31156 transcript.

(C) Conservation of the cryptic 5' SS (NNSPLICE score of 0.54) and a potential 3' recursive splice site (NNSPLICE score of 0.98 ) found on the CG31156 transcript highlighted in green, relative to the gene model. Conservation of the splice site is depicted using the multiple alignment format on the UCSC genome browser, as well as phyloP scores. Canonical splice sites are highlighted in yellow.

(D) Model of activation of dual cryptic splice sites on the CG31156 transcript. Activation of the cryptic 5' SS with an additional cryptic 3' recursive splice sites leads to deletion of $110 \mathrm{nt}$ of mRNA. 
A baboon (Uniprot: A1Z7L9)

bioRxiv preprint doi: https://doi.org/10.1101/20280733231498; this version postedulyisordered

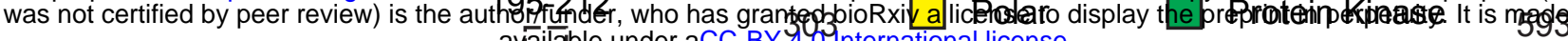

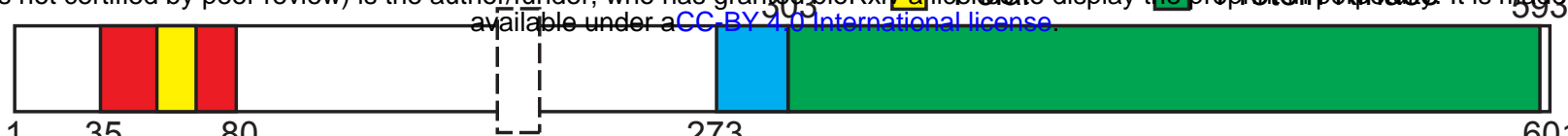

1. Disulfide bond b/w 180 and 194

2. Transmembrane $224-246$
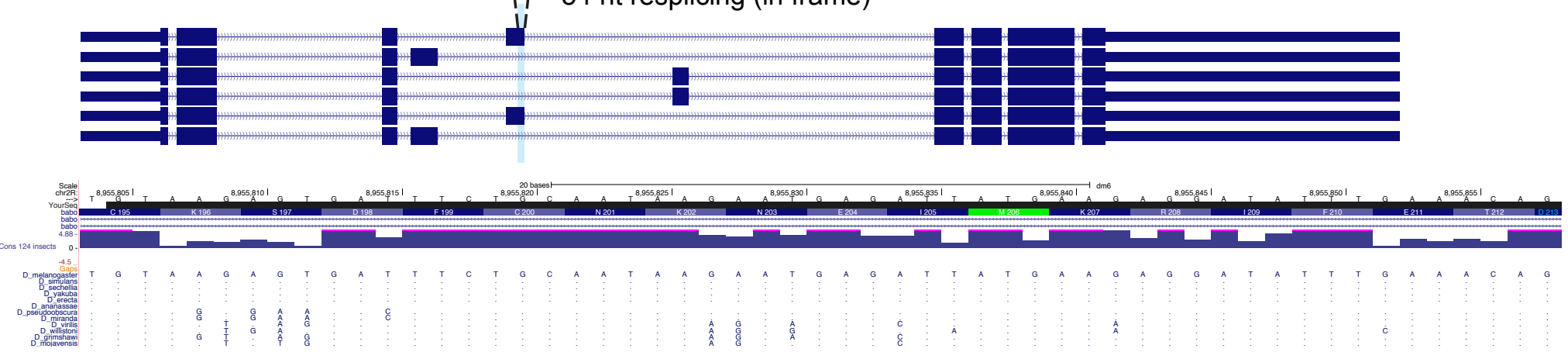

B elF4G1 (Uniprot: O61380)

MI (after MA-3 and elF4G)

W2 $1300 \quad 1433$

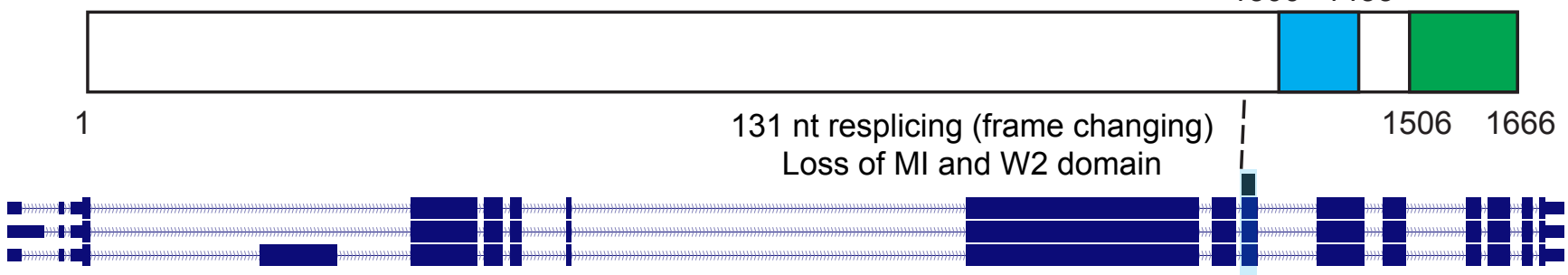

C straw (Uniprot: A1Z6F6) $\quad \square$ Plastocyanin-like

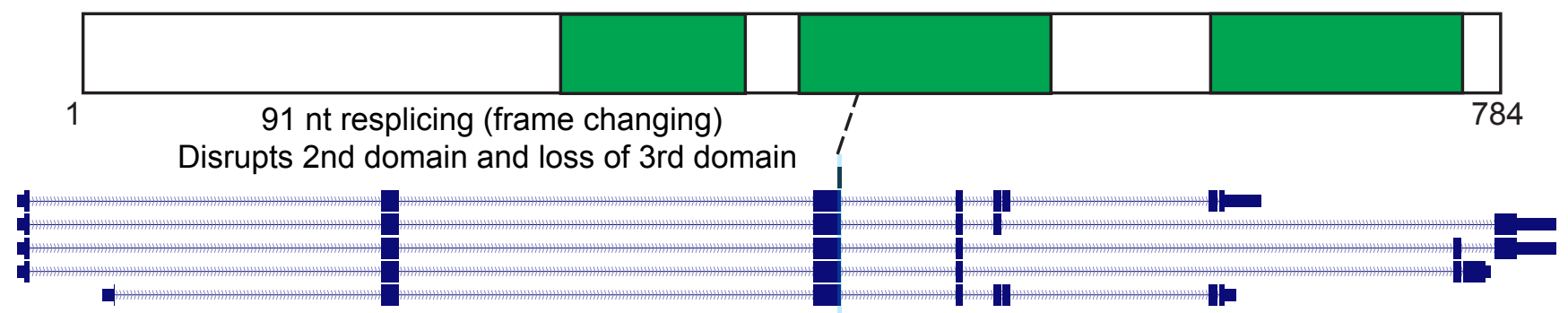

Figure 4 - figure supplement 3. Re-splicing on mRNAs alters translated proteins

(A-C) Protein and transcript structures are schematized and the location of cryptic resplicing highlighted in blue.

(A) Re-splicing on baboon leads to a 54 nt deletion of the mRNA and an 18 amino acid deletion. The deletion does not overlap known domains. Conservation plots for deleted $54 \mathrm{nt}$ region is included.

(B) Re-splicing on elF4G1 leads to a $131 \mathrm{nt}$ deletion, leading to a change in reading frame and truncation of the $\mathrm{C}$ terminal domains of elF4G1. Importantly, critical domains required for elF4G1 function are lost due to re-splicing.

(C) Re-splicing on straw leads to a $91 \mathrm{nt}$ deletion, leading to a change in reading frame and truncation of the protein. Importantly, 2 of 3 Plastocyanin-like domains are lost due to transcript defects. 